\title{
Bio-Inspired Nanocomposite Assemblies as Smart Skin Components
}

Xiaoyin Xiao, Amalie L. Frischknecht, Gabriel A. Montaño, Amy Allen, Thayne L.

Edwards, Komandoor E. Achyuthan, David R. Wheeler, and Susan M. Brozik

Prepared by

Sandia National Laboratories

Albuquerque, New Mexico 87185 and Livermore, California 94550

Sandia National Laboratories is a multi-program laboratory managed and operated by Sandia Corporation, a wholly owned subsidiary of Lockheed Martin Corporation, for the U.S. Department of Energy's National Nuclear Security Administration under contract DE-AC04-94AL85000.

Approved for public release; further dissemination unlimited. 
Issued by Sandia National Laboratories, operated for the United States Department of Energy by Sandia Corporation.

NOTICE: This report was prepared as an account of work sponsored by an agency of the United States Government.

Neither the United States Government, nor any agency thereof, nor any of their employees, nor any of their contractors, subcontractors, or their employees, make any warranty, express or implied, or assume any legal liability or responsibility for the accuracy, completeness, or usefulness of any information, apparatus, product, or process disclosed, or represent that its use would not infringe privately owned rights. Reference herein to any specific commercial product, process, or service by trade name, trademark, manufacturer, or otherwise, does not necessarily constitute or imply its endorsement, recommendation, or favoring by the United States Government, any agency thereof, or any of their contractors or subcontractors. The views and opinions expressed herein do not necessarily state or reflect those of the United States Government, any agency thereof, or any of their contractors.

Printed in the United States of America. This report has been reproduced directly from the best available copy.

Available to DOE and DOE contractors from

U.S. Department of Energy

Office of Scientific and Technical Information

P.O. Box 62

Oak Ridge, TN 37831

Telephone: (865)576-8401

Facsimile: $\quad$ (865) $576-5728$

E-Mail: $\quad$ reports@adonis.osti.gov

Online ordering: http://www.osti.gov/bridge

Available to the public from

U.S. Department of Commerce

National Technical Information Service

5285 Port Royal Rd

Springfield, VA 22161

Telephone: $\quad$ (800)553-6847

Facsimile: (703)605-6900

E-Mail: $\quad$ orders@ntis.fedworld.gov

Online order: http://www.ntis.gov/help/ordermethods.asp?loc=7-4-0\#online

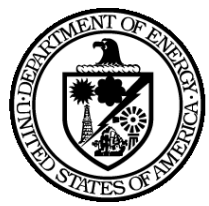


SAND2011-6839

Unlimited Release

Printed October 2011

\title{
Bio-Inspired Nanocomposite Assemblies as Smart Skin Components
}

Xiaoyin Xiao, Amalie L. Frischknecht, Gabriel A. Montaño, Amy Allen, Thayne L.

Edwards, Komandoor E. Achyuthan, David R. Wheeler, and Susan M. Brozik

Sandia National Laboratories

Albuquerque, NM 87185

\begin{abstract}
There is national interest in the development of sophisticated materials that can automatically detect and respond to chemical and biological threats without the need for human intervention. In living systems, cell membranes perform such functions on a routine basis, detecting threats, communicating with the cell, and triggering automatic responses such as the opening and closing of ion channels. The purpose of this project was to learn how to replicate simple threat detection and response functions within artificial membrane systems. The original goals toward developing "smart skin" assemblies included: 1) synthesizing functionalized nanoparticles to produce electrochemically responsive systems within a lipid bilayer host matrices, 2) calculating the energetics of nanoparticle-lipid interactions and pore formation, and 3) determining the mechanism of insertion of nanoparticles in lipid bilayers via imaging and electrochemistry. There are a few reports of the use of programmable materials to open and close pores in rigid hosts such as mesoporous materials using either heat or light activation. However, none of these materials can regulate themselves in response to the detection of threats. The strategies we investigated in this project involve learning how to use programmable nanomaterials to automatically eliminate open channels within a lipid bilayer host when "threats" are detected. We generated and characterized
\end{abstract}


functionalized nanoparticles that can be used to create synthetic pores through the membrane and investigated methods of eliminating the pores either through electrochemistry, change in $\mathrm{pH}$, etc. We also focused on characterizing the behavior of functionalized gold NPs in different lipid membranes and lipid vesicles and coupled these results to modeling efforts designed to gain an understanding of the interaction of nanoparticles within lipid assemblies. 


\section{CONTENTS}

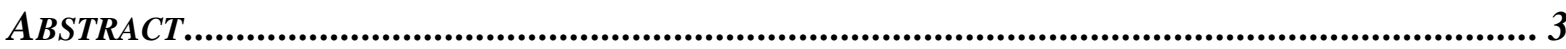

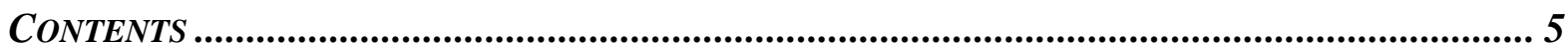

1 Theoretical modeling of insertion of a nanoparticle into lipid bilayers..............

1.0 Preliminary calculations

1.1 Introduction: optimizing coarse-grained models of lipid bilayers

1.2 Methods

1.3 Coarse-grained models of DPPC

1.4 Results

1.4.1 State point and solvent parameters

1.4.2 Hydrocarbon models

1.4.3 Head group models

1.5 Conclusions

1.6 References

2 Surface charge directed lipid bilayer disruption and nanoparticle deposition...... $\quad .26$

2.1 Introduction

2.2 Materials and Methods

2.3 Results and Discussion

2.3.1 Nanostructured lipid bilayers

2.3.2 Dependence of bilayer surface charge density on membrane disruption and particle deposition

2.3.3 Influence of QDs surface charge on membrane disruption and particle deposition Acid induced bilayer disruption and budding.

2.4 Conclusions

2.5 References

3 Lipid bilayer templated gold nanoparticles nanoring formation using zirconium ion coordination chemistry ..................................................................40

3.0 Introduction

3.1 Materials and Methods

3.2 Results and Discussion

3.2.1 AuNPs assembly 
3.2.2 Zirconium ion induced nanoring formation

3.2.3 Acid induced bilayer disruption and budding

3.2.4 proposed nanoring formation mechanism

3.3 Conclusions

3.4 References 


\section{Theoretical modeling of insertion of a nanoparticle into lipid bilayers}

\subsection{Preliminary calculations}

The original goal for modeling was to calculate the free energy for insertion of a nanoparticle into a lipid bilayer, the thermodynamics of nanoparticle aggregation and pore formation, and the effects of lipid composition on these processes. To do so requires three-dimensional calculations using a coarse-grained model for the experimental system. A promising theoretical technique is fluids density functional theory, which is an equilibrium theory based on the statistical mechanics of fluids. Sandia's state-of-the-art molecular modeling code Tramonto is the only fluids density functional theory (DFT) code available which is capable of performing these kinds of computational studies.

We used Tramonto to perform some initial calculations on nanoparticles in single-component lipid bilayers. There are two essential ingredients in our modeling of lipid bilayers: the particular version of DFT that we use and the coarse-grained model used to describe the lipid molecules and the water. In previous work, we had developed a simple coarse-grained model of a single-component lipid bilayer, and studied it using the Chandler-McCoy-Singer DFT. ${ }^{1}$ As explained in more detail below, a more recent and modern DFT, known as the "modifiediSAFT" approach, ${ }^{2}$ has been developed and implemented into Tramonto. This new version includes a more accurate description of the single site solvent we are using to model water, compared to previous F-DFTs we have used. For our initial calculations, we used both flavors of DFT.

To model the lipids, we initially used our previous coarse-grained model in which the lipid has 2 head group sites of diameter $\sigma_{h}=1.44 \sigma$ and two tails with 8 sites each of diameter $\sigma_{\square}=\sigma$, representing the tail groups. ${ }^{3}$ Water is represented by a single site, also of diameter $\sigma_{s}=\sigma$. In the original work with the CMS functional, the different sites interacted with Lennard-Jones potentials:

$$
u_{i j}(r)=4_{i j} \quad \frac{{ }^{i j}}{r} \div \frac{{ }^{12}}{r} \div
$$


We assumed all interactions had the same interaction energy $\varepsilon$, and used a cutoff of $3.5 \sigma$ beyond which $u_{i j}(r)=0$. For purposes of this document, we refer to this model as Model A. We note that the lipid bilayer profile corresponding to Model A but calculated with modified iSAFT agrees much better with molecular dynamics (MD) simulations ${ }^{4}$ on the same model than did the original CMS functional. The comparison is shown in Fig. 1 on the left.

We next reparameterized the energetic interactions between the lipid sites to more closely mimic experimental systems. In particular, we determined appropriate solvent-solvent interactions to mimic water at $40 \mathrm{C}$ and atmospheric pressure, and then determined the tail group (hydrocarbon) energies by comparison with the partitioning free energy for hexadecane in water. The resulting interactions are $\varepsilon_{t t}=1.0, \varepsilon_{h t}=0.22=\varepsilon_{t s}=0.22$, and $\varepsilon_{h h}=$ $\varepsilon_{s s}=\varepsilon_{h s}=1.43$, all in units of the thermal energy $k T$. We will denote this bilayer model as Model B. Fig. 1.1 (right) shows the density profile along the direction normal to the lipid bilayer for our Model B. The points are again from MD simulations and the curves are from the modified iSAFT DFT. The agreement is good but not as good as for the bilayer of our original model. Note that the original Model A corresponds to a higher temperature of $k T / \varepsilon_{\square \square}=1$, whereas Model B has $k T / \varepsilon_{\square \square}=0.7$.
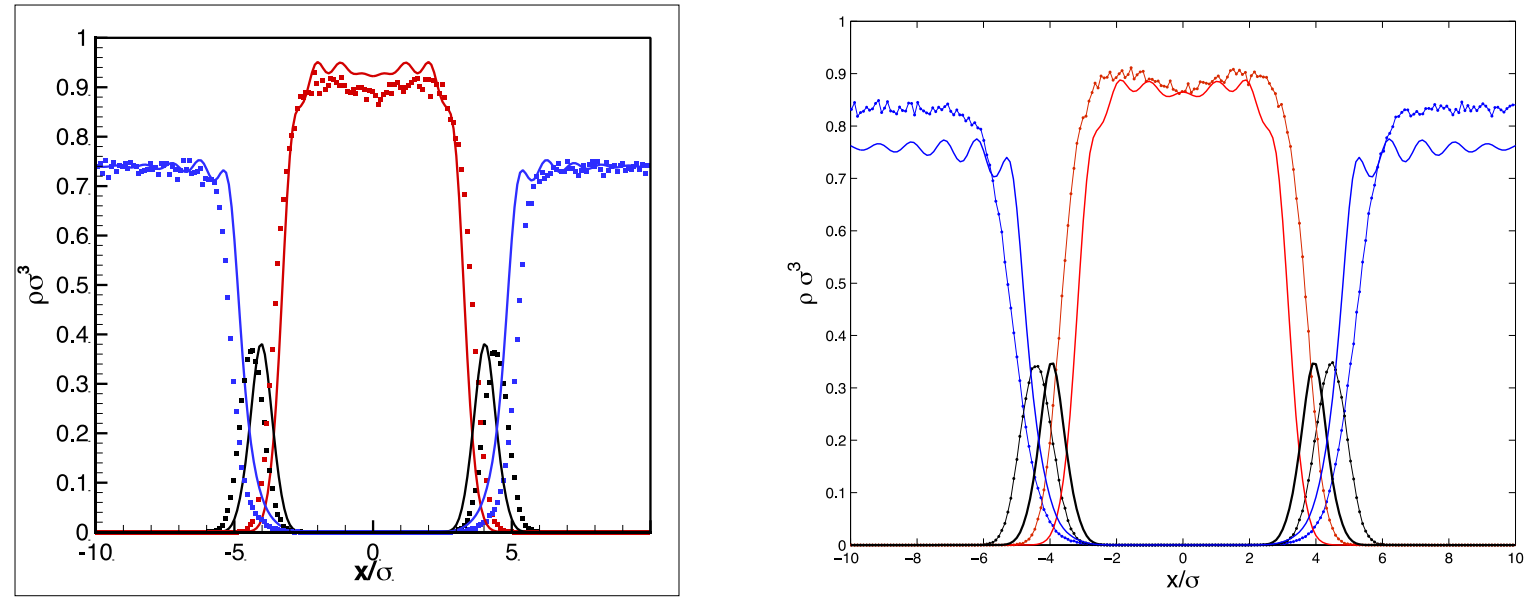

Figure 1.1. Comparison of lipid bilayer profiles calculated using the modified iSAFT DFT (solid curves) and MD simulations (points), for: left, a bilayer with all $\varepsilon / k T=1$ (Model A); right, a bilayer with $\varepsilon_{t t}=1.0, \varepsilon_{h t}=0.22=\varepsilon_{t s}=0.22$, and $\varepsilon_{h h}=\varepsilon_{s s}=\varepsilon_{h s}=1.43$ (Model B). 
The curves show tail densities (red), head group densities (black), and solvent densities (blue).

We next investigated the insertion of nanoparticles into the lipid bilayers, modeling the particles as small hard spheres of diameter $D=3 \sigma$. The insertion free energy is defined as the difference in free energy for the nanoparticle to be in the center of the bilayer, relative to the free energy of the bilayer with no nanoparticle present: $\Omega_{\mathrm{i}}=\Omega_{\mathrm{np}}-\Omega_{\text {bilayer }}$. Surprisingly, we found qualitatively different results for the free energy of insertion into a Model A bilayer calculated using the original CMS DFT, compared to bilayers of Model B calculated with the modified iSAFT DFT. Inserting a hard nanoparticle into the Model A CMS bilayer, we find that the insertion free energy is negative, $\Omega_{\mathrm{i}}=-66.9 \mathrm{kT}$, implying that it is favorable to insert a nanoparticle into the bilayer. This is somewhat surprising, since the bilayer must deform to accommodate the nanoparticle, and additionally there are no attractive interactions between the particle and the bilayer. By contrast, the insertion free energy for a nanoparticle in a Model B, modified iSAFT bilayer is $\Omega_{\mathrm{i}}=58.9 \mathrm{kT}$, so that it costs a significant amount of energy to insert the hard nanoparticle into the bilayer. Intuitively this seems more reasonable. The density profile of the lipid tails around the inserted nanoparticle is shown in Fig. 1.2 (left). We found that introducing an attractive interaction with the lipid tails to create a hydrophobic nanoparticle reduces the free energy cost, as shown in Fig.1.2 (right). Finally we found that we could not numerically converge systems with nanoparticles larger than $D=3 \sigma$ in the lipid bilayers, for either model. 

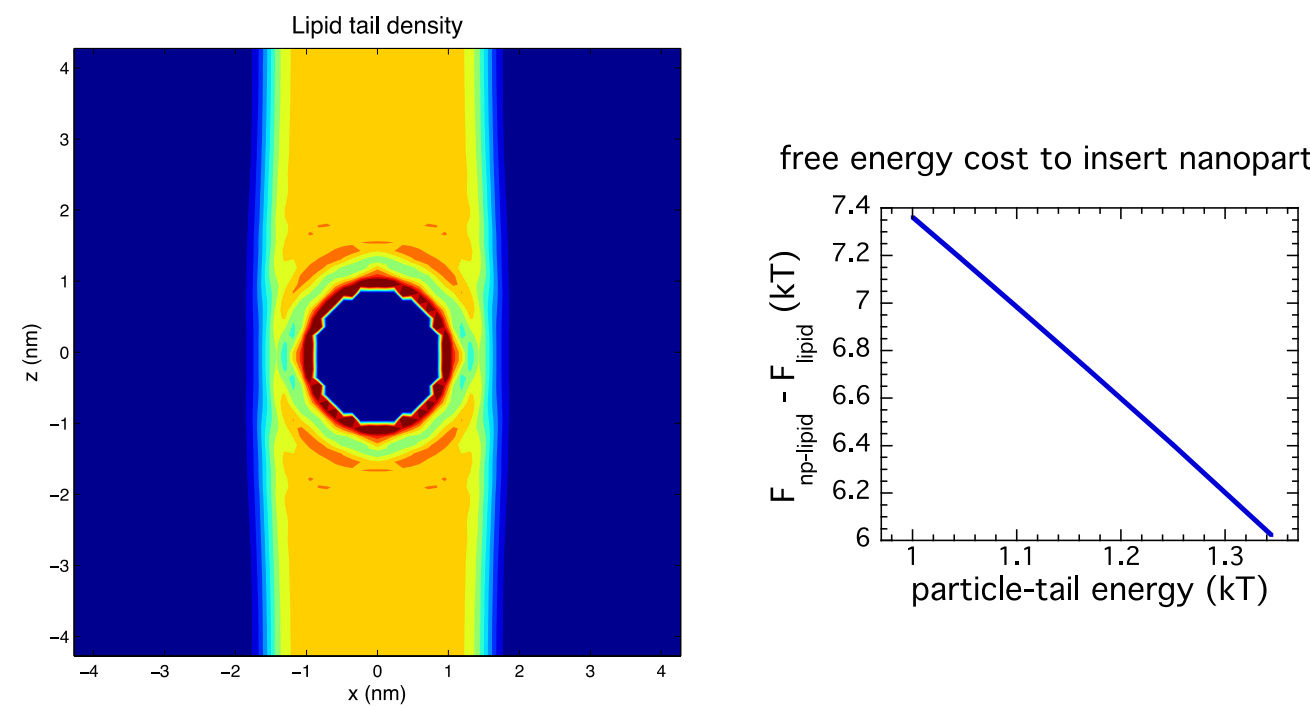

Figure 1.2. Results for inserting a hard nanoparticle into a Model B bilayer. Left: density of tail segments around the nanoparticle. Right: free energy cost to insert the particle as a function of the particle-tail interaction energy.

A preliminary conclusion of this work is that the CMS functional is not appropriate for studying nanoparticle-lipid complexes. Also, in part due to the discrepancies between the MD and modified iSAFT DFT results for Model B (Fig. 1, right), we decided to perform a more careful optimization of the lipid parameters. The remainder of this chapter describes a systematic coarse-graining of a lipid bilayer model, using the modified iSAFT DFT.

\subsection{Introduction: Optimizing Coarse-grained Models of Lipid Bilayers}

Coarse-grained modeling of biological systems has become more widely used in recent years. The interest in coarse-grained (CG) models stems from the terrific cost associated with treating these complex fluid systems with classical all-atom molecular dynamics (MD) simulations. Lipid bilayers in particular have been studied with a diverse range of models and methods (theory and simulation). Early MD simulations employed fairly generic CG lipid models; $;{ }^{5,6}$ more recent efforts have included extensive fitting of CG models for lipids based on experiment and/or atomistic MD simulations in order to obtain realistic bilayer properties from CG simulations. ${ }^{7-16}$ 
Statistical mechanical theories have also been used to study coarse-grained models of lipids. Self-consistent field theory (SCFT) has been widely applied to polymeric fluids ${ }^{17}$ and more recently to lipid bilayers. ${ }^{18,19}$ For biological membranes where the length scale of atoms is likely important, a complementary statistical mechanical approach is Fluids-Density Functional Theory (F-DFT), which differs from SCFT in that it retains information on the length scale of the CG atoms. Major advantages of using statistical theories, and in particular F-DFTs, to study lipid bilayers include less computational expense and the fact that the equilibrium free energy of the system is a natural output of the theory, unlike in molecular simulations. ${ }^{1}$ We recently applied a Fluids-DFT to model single component lipid bilayers, ${ }^{3,4}$ the effects of alcohols on lipid bilayers, ${ }^{20}$ and the formation of pores in assemblies of simple $\alpha$-helix antimicrobial peptides embedded in bilayers. ${ }^{21}$

These previous Fluids-DFT studies of biological membranes applied the Chandler-McCoySinger DFT ${ }^{1}$ for polymer fluids to a simple, generic CG lipid model. This theory is based on a second order expansion of the free energy. One limitation of the approach is that it cannot predict liquid-vapor transitions. Without access to a basic fluid phase diagram, it is impossible to clearly define the thermodynamic state of the system, and therefore difficult to rationally set coarse-grained parameters.

More recently, a novel F-DFT method (known as the "modified-iSAFT" approach) has been developed. $^{2}$ This method employs bonding constraints identical to the CMS F-DFT approach, but the modified-iSAFT approach is based on a perturbation to an atomistic reference fluid. As a result, the bonding constraints of the modified-iSAFT approach can be built on an accurate fundamental measures theory (FMT) model for the hard sphere reference fluid. $^{22}$ When FMT-based perturbative approaches are used to study attractive atomistic fluids, both bulk phase transitions and many novel phase transitions in confined media can be predicted. The extension of this approach to bonded fluids provides a new opportunity for careful parameterizations of complex fluid systems such as bilayer-forming lipids in water.

In this chapter we demonstrate the detailed application of the "modified-iSAFT" method to the optimization of a coarse-grained model of dipalmitoylphosphatidylcholine (DPPC) 
bilayers, using available experimental measures of DPPC bilayers and their constituent groups. Our coarse-graining approach is similar to that used in development of the MARTINI force field. ${ }^{8,10}$

\subsection{Methods}

This work is based on a particular fluids-DFT approach known as the modified-iSAFT method. A derivation of the theory can be found elsewhere. ${ }^{2}$ In modified i-SAFT the reference fluid consists of a simple fluid of hard spheres, which are the sites that make up the molecules of interest. The bonding constraints are treated as a perturbation to this reference hard sphere fluid. Attractions between the sites are also included as a perturbation. This Fluids-DFT approach starts with a definition of the grand free energy $\Omega$, which is a functional of the densities of each fluid component $\rho_{\alpha}(\mathbf{r})$. This functional obeys a variational principle, in which the equilibrium density profile minimizes the functional and results in the grand potential free energy of the system. Performing this minimization leads to a set of nonlinear integral equations that are solved for the density profiles.

The system of nonlinear integral equations that make up the modified i-SAFT theory are solved using the open source software, Tramonto (see http://software.sandia.gov/tramonto) developed at Sandia National Laboratories. The set of residual equations associated with the modified-iSAFT approach are solved in real space on a Cartesian grid. An iterative Newton's method approach is used to solve the nonlinear problem, and the embedded linear matrix problem is solved with a segregated Schur complement approach. These algorithms work well across several classes of Fluids-DFTs on computational platforms ranging from desktop workstation platforms to distributed memory massively parallel computers. ${ }^{23}$

The optimization studies presented here rely heavily on arc-length continuation (ALC) and binodal constraint algorithms developed previously in the context of studies on porous

materials. ${ }^{24,25}$ Those approaches are based on the use of bordering algorithms for expanding the set of equations. Binodal calculations allow the simultaneous solution of density profiles in two different computational domains where the chemical potentials of all species as well 
as the free energy are held constant across both domains. These are precisely the constraints of phase equilibria.

\subsection{Coarse-grained models of DPPC}

The key property of lipids that gives rise to their ability to self assemble in water is their amphiphilic nature. Typical phospholipids consist of a polar segment (the head group), connected to two hydrocarbon chains which are nonpolar in character (the tail group). When this molecule is submerged in a polar solvent (water), the head groups form an interface between a nonpolar region rich in tail groups and the polar solvent. Depending on the precise geometry of the molecules, lamellar phases, hexagonal phases, or other complex phases such as the bicontinuous phase may form. In biological systems, the lamellar phase is the most important since lipid bilayers form the membranes of cells. This work will focus on a common model lipid, DPPC. Its structure is shown in Fig. 1.3.

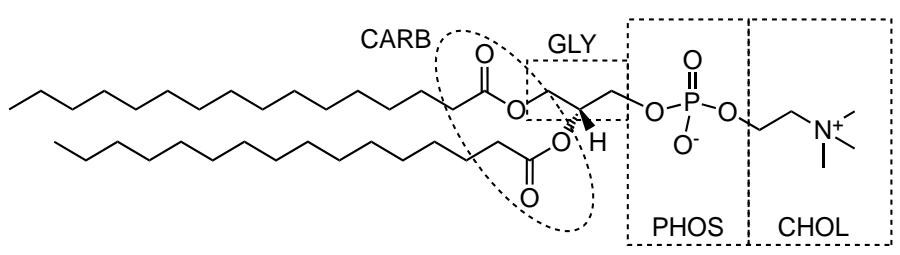

Figure 1.3. Chemical structure of DPPC, along with a definition of various parts of the head groups.

In virtually all CG models of lipids, the molecule will be split into hydrophobic and hydrophilic parts. Those parts will often be simplified into connected beads with uniform properties. Any CG model of a lipid bilayer also requires some treatment of the surrounding solvent (usually an electrolyte). The simplest CG solvent is a continuum model (implicit solvent) where the solvent has no discrete size and may or may not have a defined dielectric constant.

In this study, DPPC is treated as either a 2-bead or a 4-bead head group connected to a tail that is composed of two strands of CG hydrocarbon beads. Each tail strand is 4, 8, or 16 beads long. The solvent is modeled as a single site that may represent one to four water 
molecules. Note that the $16 \mathrm{mer}$ tails are similar to a united atom type of approach, the 8mer tails are similar to our previous work on CG lipids, ${ }^{3}$ and the 4-mer tails most closely correspond to work related to the MARTINI force field. ${ }^{8,10}$ In this work, all bonds have a fixed length and the beads in the lipid are tangent to each other and freely jointed, with no angle or dihedral interactions. This freely-jointed chain model for the lipids typically precludes the presence of a gel transition, but is adequate for describing lipid bilayers in the fluid phase. ${ }^{3,4}$

The optimizations presented here include tail and head group architecture as well as the interaction potential parameters needed to define the model. Optimizations are based on known experimental measures for water, water-hexadecane interfaces, and DPPC bilayers. The nonbond CG potentials are of a Lennard-Jones form:

$$
u_{i j}(r)=4_{i j} \quad \frac{{ }^{i j}}{r} \div \frac{{ }^{i j}}{r} \div
$$

Electrostatics are not explicitly included. This approach assumes that electrostatic effects are highly screened and that in a coarse-grained sense, potentials are not dominated by the $1 / r$ character of electrostatic interactions.

Even with this simple model, there are twelve independent $\varepsilon_{\mathrm{ij}}$ and $\sigma_{\mathrm{ij}}$ parameters as well as two state parameters (temperature, $T$, and pressure, $p$ ) that must be rationally set for this rather simple 3 component (head/tail/solvent) CG model of a lipid/solvent system. We reduce this number to nine molecular parameters by setting the cross terms $\sigma_{\mathrm{ij}}=\left(\sigma_{\mathrm{ii}}+\sigma_{\mathrm{jj}}\right) / 2$. Here the solvent-solvent parameters will be written without indices, $\sigma=\sigma_{\mathrm{ss}}$ and $\varepsilon=\varepsilon_{\mathrm{ss}}$.

\subsection{Results}

The overall strategy for optimization of the CG model is to start with the simplest component of the system, and then build complexity. So, the solvent model is optimized first, followed by optimization of the hydrocarbon tails and the water-hydrocarbon interface. Finally we consider known properties of DPPC bilayers to optimize the CG head groups in the model. 


\subsubsection{STATE POINT AND SOLVENT PARAMETERS}

A single site LJ model is used for the solvent regardless of whether it represents 1, 2, or 4 water molecules. For the atomic (or 1-mer) LJ model, there is only one bulk phase diagram in reduced units. The thermodynamic state of the CG model system is set using the phase diagram of water as a guide. Specifically, we require that the pure solvent is in the liquid state near the triple point. This constraint reflects the state of water at physiological conditions $(310 \mathrm{~K})$ relative to the water phase diagram (critical point $647 \mathrm{~K}$; triple point $273 \mathrm{~K})$. The triple point of the $\mathrm{LJ}$ fluid is $k T / \varepsilon \approx 0.65$ while the critical point is $k T / \varepsilon=1.35$ where $k$ is the Boltzmann constant (we also define $\beta=1 / k T$ ). Given the reduced triple and critical points, a reduced temperature of $k T / \varepsilon=0.68$ is chosen for all calculations. Energetics will be treated only in reduced units so both $T$ and $\varepsilon$ are set by this choice.

Setting the density of the CG solvent is more ambiguous than the temperature both because the liquid-state densities of the LJ model fluid are systematically lower than water, and because the effective density, $\rho \sigma^{3}$, is very sensitive to the effective size of the solvent $\sigma$. Using the relative pressure as a guide (water at $310 \mathrm{~K}$ has a relative pressure of $p / p_{\text {sat }}=1$ atm $/ 0.0728 \mathrm{~atm}=13.74)$, we set the CG fluid density to $\rho \sigma^{3}=0.8656$ where $\left.p \sigma^{3} / k T\right) / p_{\text {sat }}$ $=0.01715 / 0.001248=13.74$. All subsequent calculations are carried out at a pressure of $p \sigma^{3} / k T=0.0175$.

The specific value of $\sigma$ in the CG solvent model depends on the number of water molecules that are represented by the CG site. A simple volumetric scaling is used to determine $\sigma$ for three different models. Table 1.1 provides the values of $\sigma$ for cases M1, M2, and M4 that correspond to 1 water molecule, 2 water molecules, or 4 water molecules per CG site. 
Table 1.1: Size of solvent particles in various CG models.

\begin{tabular}{|l|l|l|l|}
\hline Model & \# waters per bead & CG solvent volume & $\sigma(\mathrm{nm})$ \\
\hline M1 & 1 & $\mathrm{~V}_{1}$ & 0.315 \\
\hline M2 & 2 & $2 \mathrm{~V}_{1}$ & 0.397 \\
\hline M3 & 4 & $4 \mathrm{~V}_{1}$ & 0.500 \\
\hline
\end{tabular}

\subsubsection{HYDROCARBON MODELS}

The second constituent group of the lipid system that can be optimized without consideration of the entire lipid molecule is the hydrocarbon tail group. Hexadecane is most similar to the tail groups of DPPC, and is used as a basis for the optimization. Three CG models of hexadecane are considered: a 16 bead chain, a 8 bead chain, and a 4 bead chain. These models are combined with the solvent models, M1, M2, and M4 respectively. Thus the hydrocarbon chains and water molecules are coarse-grained consistently in each of the models.

For all of the models, we set the size of the CG hydrocarbon beads $\sigma_{t t}=\sigma$. This choice is justified by a consideration of the molecular volume of hexadecane, estimated to be $28.1 \mathrm{~nm}^{3}$ from QSAR calculations. Using this volume, the CG hydrocarbon diameter is found to be $\sigma_{t t}$ $=1.02 \sigma$ for all models. Neglecting the $2 \%$ difference, the hydrocarbon and solvent sizes are set to be identical. Note that these sizes do not correspond to modern nonbonded potential parameters (e.g. $\sigma_{t t}=0.396 \mathrm{~nm}$ for $\mathrm{CH}_{2}$ groups in the TraPPE potential). The discrepancy lies in the fact that the current model is based on tangent spheres while the TraPPE and other united atom approaches are based on overlapping bonded spheres.

The energy parameter $\varepsilon_{t t}$ for the hydrocarbon groups is set by considering the expected bulk liquid density of hexadecane compared to water at our chosen pressure of $p \sigma^{3} / k T=0.0172$. Since the CG solvent density is set to $\rho \sigma^{3}=0.8656$, and hexadecane is expected to have a similar, but slightly lower density than water, we choose to set $\rho \sigma_{t t}{ }^{3}=0.85$. The value of $\varepsilon_{t t}$ is 
then found from the isotherm $k T / \varepsilon_{t t}$ where $\rho \sigma_{t t}{ }^{3}=0.85$ at the desired pressure. For the three model systems, we find optimal solutions when $\varepsilon_{t t} / k T=0.96(\mathrm{M} 1), \varepsilon_{t t} / k T=1.0(\mathrm{M} 2)$, and $\varepsilon_{t t}$ $/ k T=1.06(\mathrm{M} 4)$.

\subsubsection{OPTIMIZING SOLVENT-HYDROCARBON INTERACTIONS}

Two experimental measures of the interaction between water and hexadecane were used to optimize CG solvent-hydrocarbon interactions. Water and hexadecane do not mix, but rather form two nearly pure phases with a liquid-liquid interface. Both the interfacial tension, $\gamma$, of the water-hexadecane interface, and the partition coefficient, $\theta$, for hexadecane in water are known. The partition coefficient effectively measures the solubility of hexadecane in water. Experimental benchmarks are $\gamma=53.5 \mathrm{mN} / \mathrm{m}^{26}$ and $\theta=\Delta \mathrm{G} / \mathrm{kT}=-9.69 .^{27}$

Two distinct types of calculations were performed to determine $\gamma$ and $\theta$. Calculation of partition coefficients requires only bulk (uniform) solutions of the DFT equations. However, the solutions of interest are those at thermodynamic coexistence between the mostly immiscible water-rich and hexadecane-rich phases. Coexistence calculations are performed by simultaneous solution of two DFT problems along with the constraint of constant free energy (or bulk pressure). Constant chemical potentials for all species are maintained across both computational domains.

Given the two coexisting solutions, it is straightforward to calculate the partition coefficient as

$$
=G / k T=\ln \left(\begin{array}{c}
C 16 \\
w
\end{array} / \begin{array}{c}
s \\
w
\end{array}\right)
$$

where $\rho_{w}{ }^{C 16}$ is the density of water in the hexadecane-rich phase and $\rho_{w}{ }^{s}$ is the density of water in the water-rich phase. Note that $\Delta \mathrm{G}$ is a measure of the relative free energy of moving a solvent from a solvent rich environment into a hydrocarbon rich environment.

While $\theta$ is a property of the bulk fluids, obtaining $\gamma$ requires calculation of an inhomogeneous liquid-liquid interface. The interfacial tension is defined as

$$
=\left(\begin{array}{ll}
/ A &
\end{array}\right.
$$


where $\Omega$ is the free energy of the liquid-liquid interface, $A$ is the area, and $\Omega_{b}=-p V$ is the free energy of a bulk homogeneous fluid (either phase). Computing a liquid-liquid interfacial tension requires solving only one DFT problem. However inhomogeneous bulk boundary conditions must be applied on the two sides of the computational domain. On one side of the domain there is a water-rich phase, and on the other, a hydrocarbon-rich phase. Those boundary conditions are found using the bulk phase equilibria calculations described above.

To obtain an accurate calculation of $\gamma$, the computational domain must be large enough to fully resolve the interfacial structure while the solutions reach the bulk state at the boundaries. The calculations here were based on a domain size of $L=40 \sigma$. To ensure a stable interface, integration quadratures must be accurate enough in the bulk that the two pressures on the edges of the domain are equal. We varied the hydrocarbon-solvent interaction parameter $\varepsilon_{t s}$ and calculated the resulting values of $\gamma$ and $\theta$ for the three hydrocarbon models. While a very good fit ( $<10 \%$ error) to both $\theta$ and $\gamma$ can be achieved for the 8 mer CG model, the 4-mer and 16-mer models can only be optimized to within $25 \%$ error of the experimental values. When optimized, the 16-mer model has $\gamma$ and $\theta$ values that are too large while for the 4-mer model, they are too small. Considering the large simplifications of the theory and the $\mathrm{CG}$ nature of the models, this fit is adequate and provides a benchmark for improved CG modeling in the future. Table 1.2 summarizes the optimal parameters that are used in CG models of DPPC below.

Table 1.2: Optimized parameters for CG models of hexadecane/water systems.

\begin{tabular}{|l|l|l|l|l|}
\hline Model & $\varepsilon_{t t} / k T$ & $\varepsilon_{t s} / k T$ & $\Delta \mathrm{G} / \mathrm{kT}$ & $\gamma(\mathrm{mN} / \mathrm{m})$ \\
\hline M1 & 0.96 & 0.75 & -6.92 & 65.57 \\
\hline M2 & 1.0 & 0.6 & -9.01 & 53.23 \\
\hline M4 & 1.06 & 0.4 & -11.83 & 41.29 \\
\hline
\end{tabular}




\subsubsection{HEAD GROUP MODELS}

The DPPC head group is composed of several chemical groups as shown in Fig. 1.3. Here we consider three possible coarse-grained models for this system, as sketched in Fig.1.4. The first model (H1) splits the volume of the head group into two rather large beads. ${ }^{3}$ This model results in a linear lipid model. The second model (H2) splits the head group volume into four identical beads that are a bit smaller. Finally, model H3 has four CG beads, but two different types of beads. The head-solvent interactions of the type I and type II head group beads can therefore be varied independently. Since the larger outer beads are composed of the ionic groups PHOS and CHOL, while the smaller inner beads (type I) are composed of the CARB and GLY groups, one would expect that the type II beads could be significantly more hydrophilic than the type I beads. Sketches of three model coarse-grained head groups using the same 8-mer (model M2) tail for each case. Both the $\mathrm{H} 1$ and $\mathrm{H} 2$ models have only one type of head group bead, while the $\mathrm{H} 3$ model has two types of head group beads.

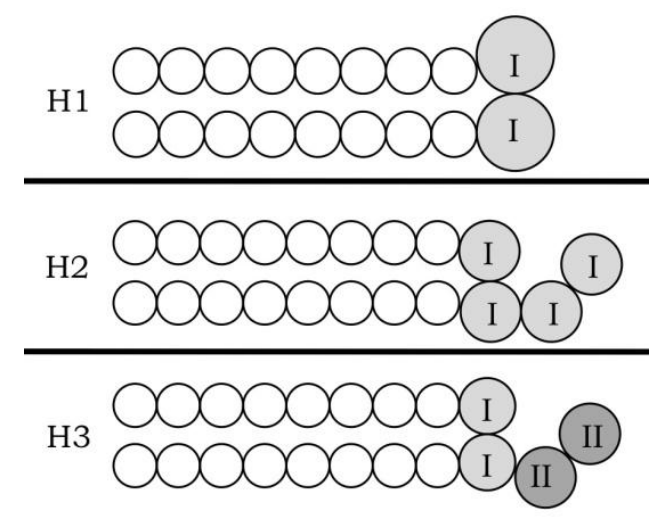

Fig. 1.4. Sketches of three model coarse-grained head groups all with the same 8-mer (model M2) tails. Both the $\mathrm{H} 1$ and $\mathrm{H} 2$ models have only one type of head group bead, while the $\mathrm{H} 3$ model has two types of head group beads.

The size of the CG beads are computed based on volumetric considerations, using the group volume data developed by Armen et al. ${ }^{28}$ (table 2 of that paper). Specifically, the total head group is taken to occupy $320 \AA^{3}$. The mean volumes for various chemical groups in the Armen study were found to be $53.7 \AA^{3}$ (phosphate group, PHOS), 108.60 $\AA^{3}$ (choline group, CHOL), 39.0 $\AA^{3}$ (carbonyl group, CARB), and $63.59 \AA^{3}$ (glycerol group, GLY). The bead sizes in each of the CG models is given in table. Note that the size of the CG head-group 
beads is independent of the solvent and tail group models (M1, M2, or M4). So any of the head group models could be coupled to any of the solvent/tail models. The head group sizes are given in Table 1.3.

Table 1.3: Particle sizes for three head group models

\begin{tabular}{|l|l|l|}
\hline Model & $\sigma_{\mathrm{h}}{ }^{\mathrm{I}}(\mathrm{nm})$ & $\sigma_{\mathrm{h}}{ }^{\mathrm{II}}(\mathrm{nm})$ \\
\hline $\mathrm{H} 1$ & 0.675 & $\mathrm{n} / \mathrm{a}$ \\
\hline $\mathrm{H} 2$ & 0.536 & $\mathrm{n} / \mathrm{a}$ \\
\hline $\mathrm{H} 3$ & 0.516 & 0.556 \\
\hline
\end{tabular}

\subsubsection{Optimizing head group interactions}

The optimization of the head group interaction parameters, $\varepsilon_{h s}$, $\varepsilon_{h h}$, and $\varepsilon_{h t}$ is based on experimental measures of DPPC bilayers. Specifically, the area per lipid $A_{L}$ and the bilayer thickness $t$ are used as benchmarks. Expected values for DPPC from the literature are $A_{L} \approx$ $61 \AA^{2}$ and $t \approx 38.3 \AA^{29,30}$

The calculation of fluid bilayer structures with F-DFT has been discussed in detail elsewhere. ${ }^{1}$ Reflecting boundaries were placed on each side of the domain with the center of the lipid bilayer at the right boundary. The full reflected bilayer is shown in the figures in this section. Only zero tension bilayers are considered in the optimization of the interaction parameters. The zero tension constraint can be expressed as

$$
=0=/ A \quad{ }_{p s} / A
$$

where $\Omega_{\mathrm{ps}}=-\mathrm{pV}$ is the free energy of the pure solvent phase.

The zero tension constraint is achieved in practice using the same binodal constraint algorithm used for calculation of partition coefficients in the previous section. One computational domain contains the bilayer while the other contains a nearly pure solvent bath (chemical equilibria demand a trace amount of lipid). The binodal algorithm enforces constant $\Omega$ between the two domains. Any of the head-group parameters $\left(\varepsilon_{h s}, \varepsilon_{h h}\right.$, or $\left.\varepsilon_{h t}\right)$ can 
be varied in the bilayer domain with virtually no impact on solvent density in the bath domain. Therefore pressure is held constant in both domains while the chemical potential of the lipid adjusts to changes in $\varepsilon$. This approach maintains a constant pressure and the zero tension constraint.

Optimization of parameters was done for all three of the head group models coupled with the 8-mer chain tail (model M2). We find that the bilayer properties are relatively insensitive to $\varepsilon_{h t}$ as compared to $\varepsilon_{h s}$ and $\varepsilon_{h h}$. Thicker, more dense bilayers are achieved when $\varepsilon_{h h}$ is increased or $\varepsilon_{h s}$ is decreased.

The H1 ( 2 bead head group) model results in bilayers that are always too thin and dilute with respect to chain packing. Specifically, $A_{L}$ is too large by at least $40 \%$ across all molecular parameter sets. In order to obtain thicker, more dense bilayers, we tried decreasing the headsolvent interactions, reflecting a shift from hydrophilic to hydrophobic head-solvent interactions. Given the polar nature of DPPC head groups, moving to a more hydrophobic CG model is not physically realistic. Therefore, we conclude that the H1 model cannot produce semi-quantitative bilayer structures. The large size of the CG head group beads in the $\mathrm{H} 1$ model relative to the solvent and tail group beads, $\sigma_{h} / \sigma=1.7$, simply prevents efficient packing of the chains in the bilayer for reasonable parameter sets.

The significant problem with the $\mathrm{H} 1$ model is corrected in both the $\mathrm{H} 2$ and $\mathrm{H} 3$ models where four smaller beads make up the head group of the lipid. In those two cases, the errors in $A_{L}$ decrease to $\geq 20 \%$ across nearly all of the molecular parameters considered. At the same time, thickness predictions improve dramatically. While $\mathrm{H} 1$ bilayers with hydrophilic head groups are found in the range $31 \AA<t<34 \AA$, the $\mathrm{H} 2 / \mathrm{H} 3$ bilayers cluster nearer the correct value with $36.5 \AA<t<39 \AA$.

Figure 1.5 shows bilayers from the $\mathrm{H} 2$ and $\mathrm{H} 3$ models. For this figure, the $\mathrm{H} 2$ parameters, $\varepsilon_{h h}$ and $\varepsilon_{h s}$, were set to be approximate averages of the $\mathrm{H} 3$ parameters (compare $\varepsilon_{h s}$ for $\mathrm{H} 2$ with $\varepsilon_{h l s}$ and $\varepsilon_{h 2 s}$ for $\mathrm{H} 3$ provided in the caption) in order to test the effect of averaging distinct chemical properties of the head groups into uniform CG beads. The parameters of the 
H3 model were chosen so the interactions of the type II head group beads (composed of the ionic CHOL-PHOS constituents) would be strong and hydrophilic. In contrast, the type I groups (composed of CARB-GLY constituents) have a more hydrophobic character. Figure 1.5 demonstrates that averaging properties among these chemical groups as in model $\mathrm{H} 2$ can yield a reasonable lipid bilayer with respect to the measures $t$ and $A_{L}$. However, in studies in which a bilayer interacts with peptides or small molecules, it may be crucial to introduce different chemical groups as in the H3 model.
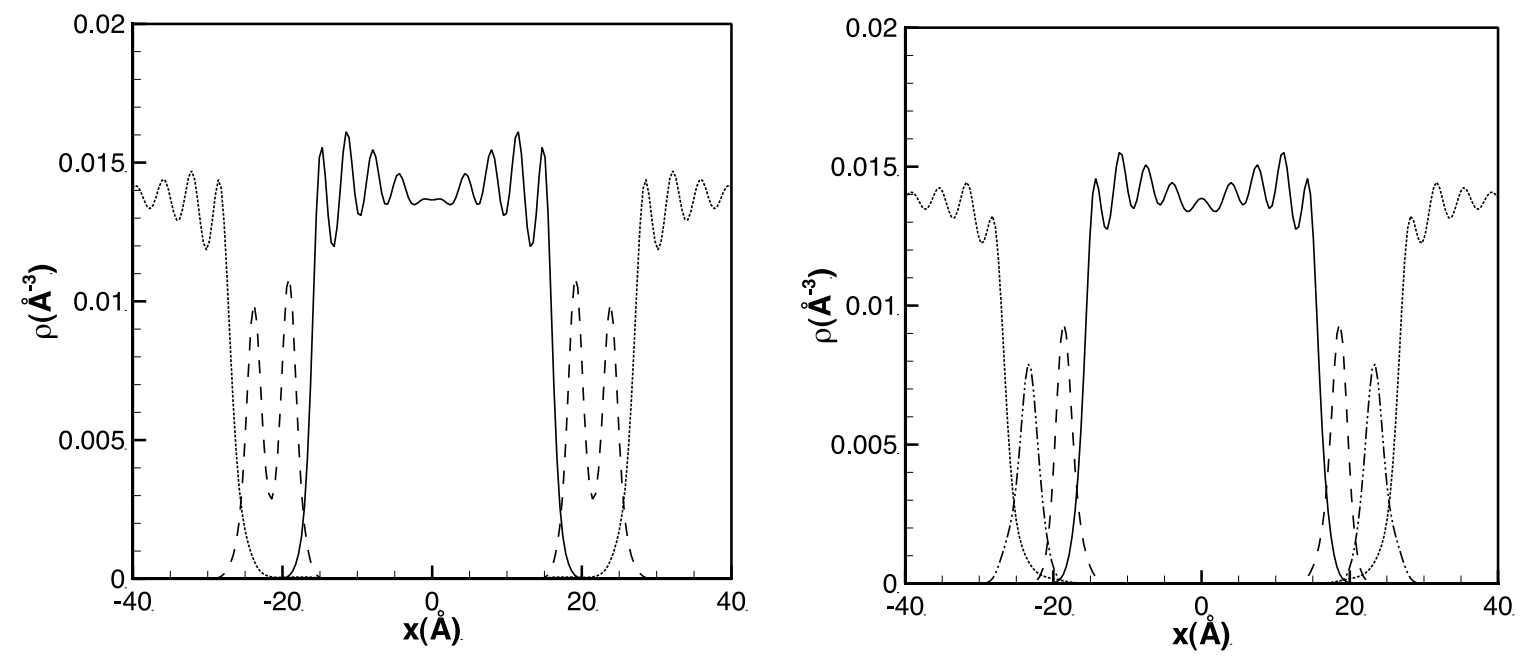

Figure 1.5. A comparison of H2 (left) and H3 (right) model bilayers where the head group properties of the uniform head model $(\mathrm{H} 2)$ are taken as approximate averages of the nonuniform head group model (H3). In both figures dotted and solid lines are solvent and tail group densities respectively. Dashed lines are head groups for model H2 and type I head groups for model H3. The dash-dot-dot line shows the type II head group for model H3.

Both the $\mathrm{H} 2$ and $\mathrm{H} 3$ model bilayers in Fig. 1.5 have about a $15 \%$ error in $A_{L}$. This could be improved by making the head groups more hydrophobic as was done for the H1 model. However, it is not physically rational to make this adjustment, and we conclude that the CG models in Fig. 1.5 have been optimized as much as is reasonable for these simple models.

Finally, we calculated lipid bilayer structures for the H2 model in combination with the 4mer chain tail (model M4) and the 16-mer chain tail (model M1). As the lipid system is 
coarse-grained, more structured packing of the tails is observed. At the same time, the bilayer thins and the area per lipid increases. The final values for the area per lipid and thickness of our optimized bilayer models, along with the experimental values, are listed in Table 1.4.

Table 1.4: Area per lipid, thickness, and are compressibility modulus for various model bilayers. The last row lists the experimental values for DPPC bilayers.

\begin{tabular}{|l|l|l|l|}
\hline Model & $A_{L}\left(\AA^{2}\right)$ & $t(\AA)$ & $K_{A}(\mathrm{mN} / \mathrm{m})$ \\
\hline H2-M4 & 77.9 & 33.0 & 325.5 \\
\hline H1-M2 & 94.7 & 32.6 & 276.3 \\
\hline H2-M2 & 69.2 & 38.3 & 436.8 \\
\hline H3-M2 & 70.4 & 37.3 & \\
\hline H2-M1 & 64.5 & 41.6 & 578.1 \\
\hline experiment & 61.0 & 38.3 & $231 \pm 20$ \\
\hline
\end{tabular}

\subsubsection{Area compressibility modulus}

As a further test of our CG models, we calculate the area compressibility modulus $K_{A}$ of the bilayers. This modulus is obtained from the dependence of the surface tension on the area per lipid near the point of zero tension:

$$
K_{A}\left(\begin{array}{ll}
A_{L} & A_{L 0}
\end{array}\right) / A_{L 0}
$$

where $A_{L O}$ is the area per lipid when $\gamma=0$. For small deviations of $A_{L}$ from its zero tension value, we find that $\gamma$ is indeed linear in $\left(A_{L}-A_{L O}\right) / A_{L O}$. Values of $K_{A}$ for the model bilayers studied are shown in Table 4. The experimental value for DPPC is $K_{A}=231 \pm 20 \mathrm{mN} / \mathrm{m} .{ }^{29}$ The F-DFT thus predicts a significantly higher compressibility modulus. However, this is to be expected because the F-DFT is an equilibrium theory which neglects the thermal fluctuations of the bilayer. These fluctuations lead to a much smaller measured value of $K_{A}$. In MD simulations, the value of $K_{A}$ is strongly system-size dependent, with larger values being found for smaller systems which have fewer fluctuation modes. Atomistic simulations on small bilayers typically report values of $K_{A}$ that are about a factor of two larger than the experimental value; this is consistent with our results. Similarly, the coarse-grained 
MARTINI model gives $K_{A}=400 \pm 30 \mathrm{mN} / \mathrm{m}$ for a small system of 256 lipids, but $K_{A}=260 \pm$ $40 \mathrm{mN} / \mathrm{m}$ for a larger system with 6400 lipids. ${ }^{8,10}$ The F-DFT should give the same result as the small MD systems, and indeed the H2-M2 model in particular is in good agreement with the simulation values for small systems.

\subsection{Conclusions}

We applied a new fluids density functional theory, the modified-iSAFT theory, to calculations of the structure and thermodynamic properties of coarse-grained models of lipid bilayers. In this chapter we have detailed methods for systematic coarse-graining of lipid molecules using experimental measures to guide the optimization of the models. The approach involves utilization of a diverse set of numerical techniques within the DFT, including high performance solvers, bifurcation algorithms, and inhomogeneous boundaries. This approach results in coarse-grained lipid bilayer models in which the properties of the underlying constituent groups (the solvent and hydrocarbons) are consistent with those of real materials. Additionally, the models result in lipid bilayers with structural and elastic properties that are consistent with experimental measures. These methods therefore provide a route to semi-quantitative CG models of lipid systems that can be treated with F-DFT.

\subsection{References}

1) A. L. Frischknecht; L. J. D. Frink; Faller, R., Jue, T., Longo, M. L., Risbud, S. H., Eds.; Humana Press: 2009; Vol. 2, p 1.

2) S. Jain; A. Dominik; W. G. Chapman J Chem Phys 2007, 127, 244904.

3) L. J. D. Frink; A. L. Frischknecht Phys Rev E 2005, 72, 041923.

4) A. L. Frischknecht; L. J. D. Frink Phys Rev E 2005, 72, 041924.

5) R. Goetz; R. Lipowsky J Chem Phys 1998, 108, 7397.

6) M. J. Stevens J Chem Phys 2004, 121, 11942.

7) J. C. Shelley; M. Y. Shelley; R. C. Reeder; S. Bandyopadhyay; M. L. Klein J Phys Chem B 2001, 105, 4464.

8) S. J. Marrink; A. H. de Vries; A. E. Mark J Phys Chem B 2004, 108, 750.

9) J. Elezgaray; M. Laguerre Comput Phys Commun 2006, 175, 264. 
10) S. J. Marrink; H. J. Risselada; S. Yefimov; D. P. Tieleman; A. H. de Vries J Phys Chem B 2007, 111, 7812 .

11) P. J. Bond; J. Holyoake; A. Ivetac; S. Khalid; M. S. P. Sansom J Struct Biol 2007, 157, 593.

12) M. Orsi; D. Y. Haubertin; W. E. Sanderson; J. W. Essex J Phys Chem B 2008, 112, 802.

13) A. Lyubartsev; A. Mirzoev; L. Chen; A. Laaksonen Faraday Discussions 2010, 144, 43.

14) W. Shinoda; R. DeVane; M. L. Klein J Phys Chem B 2010, 114, 6836.

15) Z.-J. Wang; M. Deserno J Phys Chem B 2010, 114, 11207.

16) A. P. Lyubartsev; A. L. Rabinovich Soft Matter 2011, 7, 25.

17) G. H. Fredrickson The Equilibrium Theory of Inhomogeneous Polymers; Oxford University Press: New York, 2006.

18) M. Mueller; K. Katsov; M. Schick Phys Rep 2006, 434, 113.

19) S. Pogodin; V. A. Baulin Soft Matter 2010, 6, 2216.

20) A. L. Frischknecht; L. J. D. Frink Biophys J 2006, 91, 4081.

21) L. J. D. Frink; A. L. Frischknecht Phys Rev Lett 2006, 97, 208701.

22) R. Roth J Phys-Condens Mat 2010, 22, 063102.

23) M. A. Heroux; A. G. Salinger; L. J. D. Frink Siam J Sci Comput 2007, 29, 2059.

24) A. G. Salinger; L. J. D. Frink J Chem Phys 2003, 118, 7457.

25) L. J. D. Frink; A. G. Salinger J Chem Phys 2003, 118, 7466.

26) D. M. Wu; V. Hornof Chem. Eng. Commun. 1999, 172, 85.

27) P. Schatzberg J. Phys. Chem. 1963, 67, 776.

28) R. S. Armen; O. D. Uitto; S. E. Feller Biophys J 1998, 75, 734.

29) J. F. Nagle; S. Tristram-Nagle Bba-Rev Biomembranes 2000, 1469, 159.

30) N. Kucerka; J. F. Nagle; J. N. Sachs; S. E. Feller; J. Pencer; A. Jackson; J. Katsaras Biophys J 2008, 95, 2356. 


\section{SURFACE CHARGE DIRECTED LIPID BILAYER DISRUPTION AND NANOPARTICLE DEPOSITION}

\subsection{Introduction}

Biological cell membranes are composited with unique structures and specific functionality, which includes structural domains, electrostatic charge localization, and so on. In this project, we studied the structural dependence of lipid bilayer assemblies on their composition, fluidity, and surface charges. Using three different lipids we identified unique structures and localized charge distribution which led to unique interactions with charged nanoparticles of varied surface charge densities.

In biological membranes, NPs having dimensions less than $2 \mathrm{~nm}$ can typically penetrate the cell membranes, while larger particles are mostly determined to be membrane disruptors. The membrane disruption would greatly alter the membrane permeability, membrane potential, and thus membrane functions. The surface charge of NPs plays a leading role in cell membrane disruption. ${ }^{1-5}$ It has been found that positively charged NPs disrupt cell membranes to the greatest extent, while neutral, negative, and zwitterionic NPs have negligible effects. ${ }^{6-8}$ Mammalian cell membranes, such as those of cancer cells and human airway epithelial cells, typically carry net negative charges, it is therefore the electrostatic attraction that primarily initiates membrane disruption. The experimental data are in agreement with computational modeling. ${ }^{9}$ However, when using artificial supported lipid bilayers, Banaszak Holl's group has concluded that a wide variety of positively charged NPs (AuNPs, dendrites, etc.) also disrupt neutral lipid bilayers, such as the dimyristoylphosphatidylcholine (DMPC)-containing bilayers which carry net zero charges. ${ }^{10-}$ ${ }^{12}$ Can such disruption be induced by other types of molecular interactions besides electrostatic? It is well-known that surface defects (channels, pits, cavities, etc.) can be formed during bilayer phase transition, ${ }^{13,14}$ and the fluid-to-gel phase transition of DMPC bilayers is right around room temperature. These surface defects in bilayers might play a critical role in membrane disruption. Is electrostatic interaction solely responsible for NPinduced cell membrane disruption? How do the surface charge and charge density of the NP 
and the cell membranes affect the membrane disruption and the binding of NPs to the membranes?

We sought to answer these questions by probing the NP-bilayer interactions while varying their surface charge densities. The artificial lipid bilayers were assembled from the mixtures of three lipids: cationic dioleoyltrimethylammonium propane (DOTAP), zwitterionic 1palmitoyl-2-oleoyl-sn-glycero-3-phosphocholine (POPC), and zwitterionic distearoylphosphatidylcholine (DSPC). The variation of DOTAP molar ratio in DOTAPPOPC, DOTAP-DSPC, DOTAP-DSPC-POPC two or three-lipid systems led to surface charge density (i.e., charge per surface area) changes when the bilayers were assembled on mica or glass substrates. The net positive charge is proportional to the DOTAP concentration. DOTAP carries a net positive charge, POPC and DSPC are neutral. The gold nanoparticles (AuNPs) were capped by a monolayer of 11-mercaptoundecanoic acid, resulting in a fully charged negative surface at $\mathrm{pH}$ above 8 . The partially charged AuNPs were prepared through a ligand exchange reaction between 11-mercaptoundecanoic acid and dodecanethiol. The surface charge density (i.e., charge per particle) was then varied by the molecular ratio between these two ligands adsorbed at the NP surface. The CdSe/ZnS core shell quantum dots (QDs) were functionalized with carboxylic acid terminals, and their surface charge density was varied by the $\mathrm{pH}$ value of the buffer solutions through dissociation of carboxylic acid. We used atomic force microscope (AFM) and fluorescence microscope (FL) to determine the NP-bilayer interactions through the morphology and fluorescent intensity changes in lipid bilayers before and after interacting with the NPs.

\subsection{Materials and Methods}

AuNPs Synthesis. AuNPs were prepared using published methods with minor modifications $^{15-16}$. 1-dodecanethiol coated AuNPs (i.e., AuNP-DDT) were prepared using a two-phase liquid-liquid reaction system. The fully carboxylated AuNPs (fc-AuNPs), such as 11-mercaptoundecanoic anion and 3-mercaptopropionic acid coated AuNPs (i.e., Au-MUA and Au-MPA, respectively), were prepared by the inverse microelmusion method. The partially carboxylated AuNPs (pc-AuNPs) were obtained after a ligand exchange reaction between DTT and MUA. 
Lipids and Liposomes. Lipids, DOTAP, DSPC and POPC were purchased from Avanti Polar Lipids and stored in chloroform. Liposomes were prepared by probe sonication. $20 \mathrm{mg}$ lipid powder was dissolved in $1 \mathrm{ml}$ chloroform. $76 \mu \mathrm{l}$ of such solution was dried under nitrogen stream and under house-vacuum for more than $2 \mathrm{~h}$. The lipid cake was then hydrated with N-2-hydroxyethylpiperazine-N'2-ethane sulfonate (HEPES) buffer (150 mM NaCl + 20 $\mathrm{mM} \mathrm{CaCl}_{2}+10 \mathrm{mM}$ HEPES, $\mathrm{pH}$ 7.4). After 5 freeze-thaw cycles the solution was probe sonicated for $10 \mathrm{~min}$. The size of the liposomes was fairly monodisperse around 30 to $40 \mathrm{~nm}$.

Lipid Bilayer Assembly. Supported lipid bilayers were assembled by placing liposome solutions on freshly cleaved mica or piranha (75 vol\% sulfuric acid) cleaned glass coverslips. The bilayers were first rinsed with water and then Tris- $\mathrm{HCl}$ buffer (50 mM Tris, $\mathrm{pH} 8.2)$.

AuNPs Binding. Fully and partially carboxylated AuNPs were dissolved in Tris buffer, pH 8.2, and were directly introduced onto the supported lipid bilayers by electrostatic interaction. Above $1 \mathrm{nM}$ of particle concentration, the particle coverage reached saturation.

Atomic Force Microscopy (AFM). We used Agilent Pico SPM II and MAC Type II cantilevers with a typical spring constant of $\sim 1 \mathrm{~N} / \mathrm{m}$. All images were taken in a liquid cell in MAC mode (set point, $\sim 75 \%$ of free oscillation, scan rate, $\sim 1 \mathrm{~Hz}$ ). To mechanically scratch the particle-lipid bilayer surfaces, the set point was further decreased to $\sim 30 \%$ of the free oscillation. The image analysis was performed using Gwyddion software.

Fluorescence Microscopy (FL). We used an Olympus 1X-71 inverted optical microscope to generate a TIF file of the fluorescence image and then analyzed using Gwyddion software for the scale bar, color and color scale, and optical dimensions. $0.3 \%$ of $\beta$-BODIPY ${ }^{\circledR}$ FL $_{12^{-}}$ HPC (Invitrogen, Life Technologies, Carlsbad, CA) in lipid bilayers was used. The dye had an excitation and emission maxima of 506 and 513nm, respectively.

\subsection{Results and discussion}

Figure 2.1 illustrates the structures of partially and fully charged AuNPs, CdSe/ZnS core shell QDs, and the three lipids used to assemble artificial lipid bilayers. The average core size 
of fully and partially charged AuNPs is around $3.5 \mathrm{~nm}$. The QDs are packed with carboxylic acid terminals at their outer surfaces with the hydrodynamic size of $\sim 20 \mathrm{~nm}$. We used buffer solutions with $\mathrm{pH}$ values of 5.0 to 8.2 in order to vary the amount of surface charges at the QD. At room temperature, DOTAP and POPC form miscible fluid bilayers, while DSPC has a phase transition temperature well above room temperature $\left(\sim 52^{\circ} \mathrm{C}\right)$.

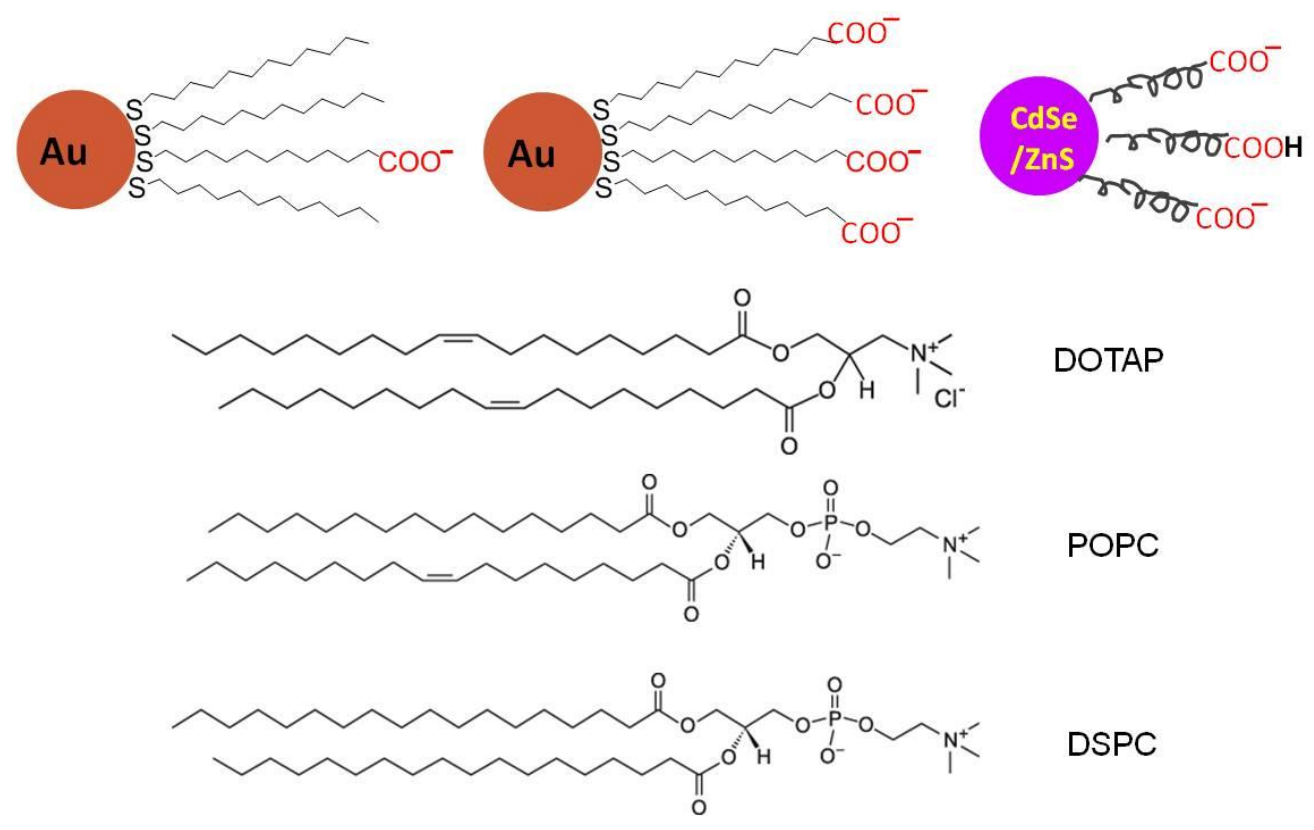

Figure 2.1. Schematics of partially and fully charged AuNPs, pH tunable negatively charged $\mathrm{CdSe} / \mathrm{ZnS}$ core shell QDs, and the three lipids used to assemble positively charged artificial bilayers.

\subsubsection{Nanostructured lipid bilayers.}

Since both DOTAP and POPC form fluidic bilayers at room temperature, their miscible mixtures, regardless of molecular ratio were also observed to be fluidic. The AFM images were nearly featureless and appeared the same for different DOTAP surface concentrations (images not shown). On the other hand, DSPC formed a bilayer gel at room temperature. The bilayer containing DSPC often showed two phases with the domain sizes varying with the DSPC concentration. ${ }^{17-19}$ Of particular interest, we found nanostructured networks within the so-called fluidic phases when the gel domains were present. Figure 2.2 shows the AFM images of DOTAP-DSPC two-lipid bilayer assembled upon freshly cleaved mica surfaces. At 
DOTAP molecular ratio of $60 \%$, as shown in Figure 2.2 (A and B), the fluidic phase occupied much more than $60 \%$ of the surface, indicating that some of DSPC molecules were mixed in DOTAP. The gel domains were not observed when the DOTAP concentration was above $85 \pm 5 \%$. The nanostructures were observed over the entire fluidic domains up to the edges of the gel domains and some of those acquired donut shapes (Figure $2 \mathrm{C}$, inset). The height variation $(<0.6 \mathrm{~nm})$ is much smaller than the height difference between the gel and the fluidic domains $(\sim 2 \mathrm{~nm})$. The high resolution images indicated three distinct structures within the fluidic phase (Figure 2D). The darkest areas, appearing as stripes or lines, were assumed as surface defects where charged DOTAP lipids were loosely packed at both the top and bottom layers. The asymmetrical distribution of DOTAP and DSPC within the bilayers resulted in brighter structures than those packed by DOTAP alone. The asymmetrical distribution of charged DOTAP lipids is useful to disperse charges and thus minimize the electrostatic repulsion among the highly charged individual domains.

The nanostructured networks were still observable after heating the bilayer to $60^{\circ} \mathrm{C}$ then cooling back to room temperature in the absence of lipid vesicles. Such thermo annealing disrupted the bilayers and led to the formation of large or small cavities near the gel domains. However, when POPC was mixed with DSPC or was added to the DOTAP-DSPC mixtures (Figure 2.3), the nanostructures could not be observed clearly or disappeared. In addition to larger gel domain sizes, the fluidic phases were featureless (Figure 2.3C, D). Since POPC is miscible with DOTAP, POPC was used to decrease the surface charge density by mixing with charged DOTAP. Such dilution of surface charges would result in decrease of the electrostatic repulsion necessary for the formation of nanostructured networks. The same mechanism also interprets the sharp decrease of gel domain sizes in the case of DSPCDOTAP bilayer mixtures. At the same percentage of DSPC, the domain sizes in DOTAPDSPC are about ten times smaller than those in POPC-DSPC and POPC-DOTAP-DSPC bilayers (Figure 2.3 and Table 2.1). The decrease of domain size with increase of surface charge density was also associated with electrostatic repulsion which must be minimized among the charged fluidic domains. ${ }^{20-23}$ 

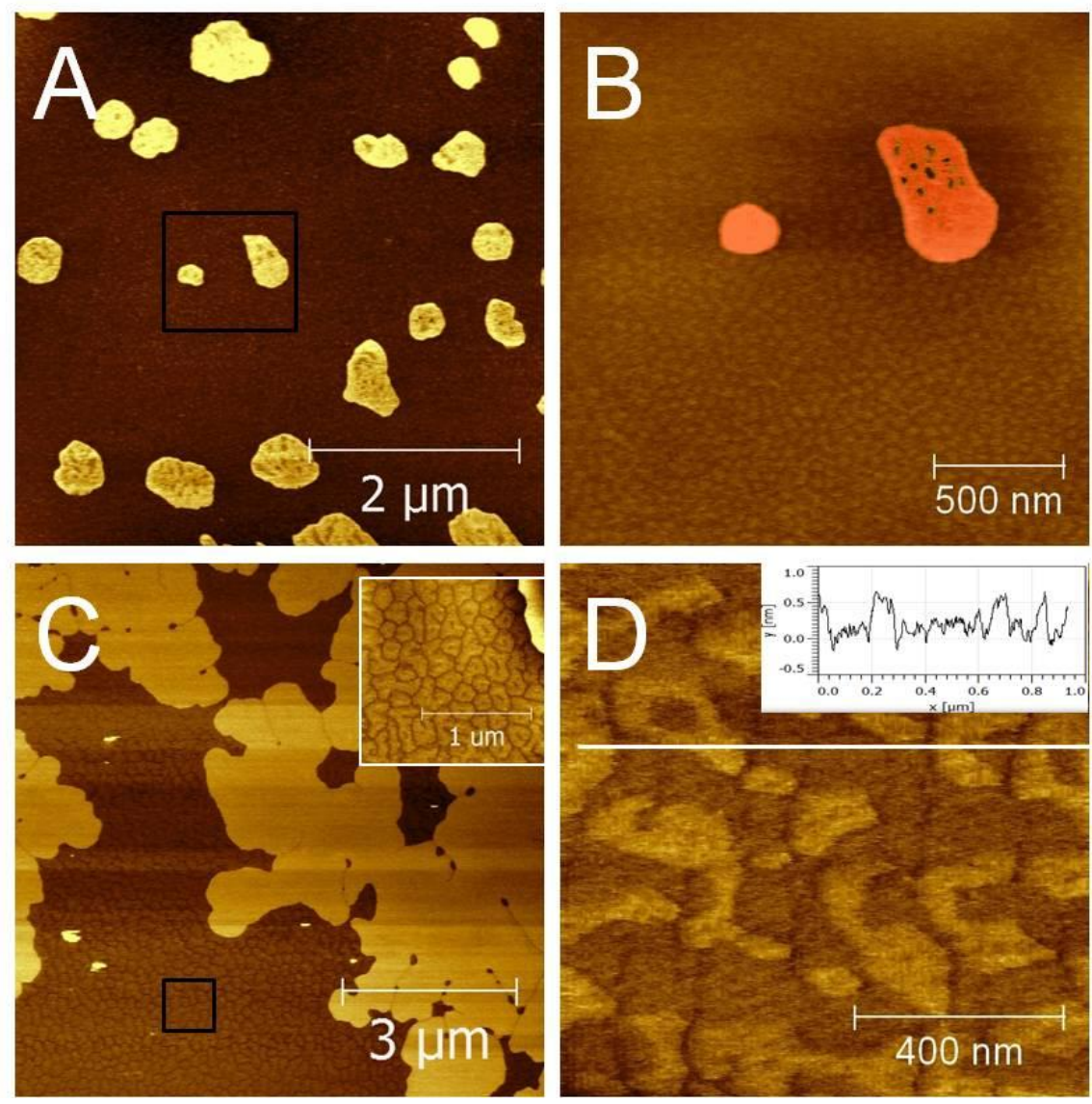

Figure 2.2 AFM images of mixed DOTAP-DSPC lipid bilayers. (A, B) 60\%DOTAP40\%DSPC, (C, D) 20\%DOTAP-80\%DSPC. 

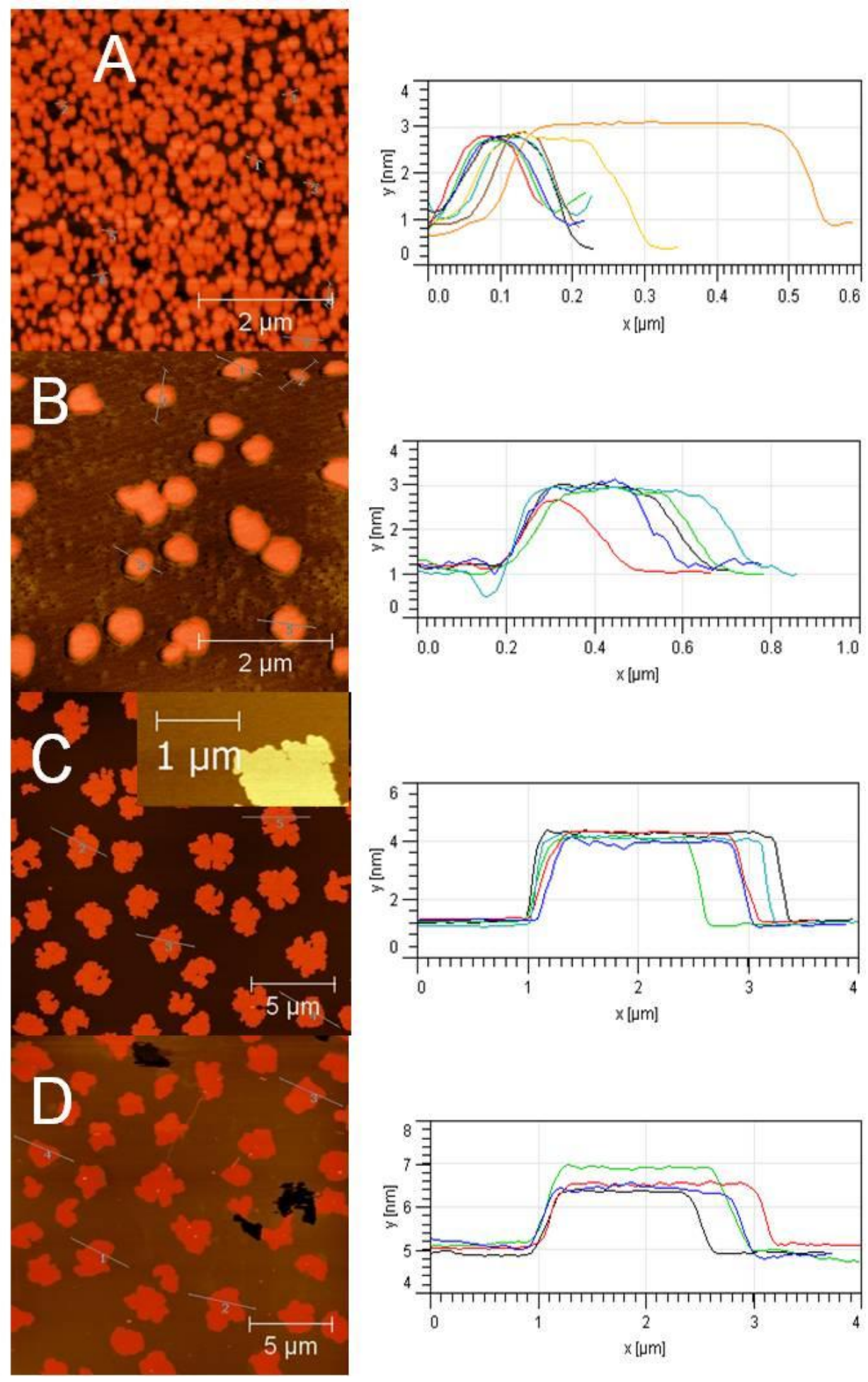

Figure 2.3. AFM images of multiple-lipid bilayers and the corresponding domain size distributions (height profiles). (A) 40\%DOTAP/60\%DSPC, (B) 60\% DOTAP/40\%DSPC, (C) $20 \% \mathrm{DOTAP} / 40 \% \mathrm{DSPC} / 40 \% \mathrm{POPC}$, (D) $40 \%$ DSPC/60\%POPC. 
Table 2.1 Effect of DOTAP concentration on gel domain size.

\begin{tabular}{|c|c|c|c|c|c|c|c|}
\hline \multirow{3}{*}{$\begin{array}{c}\text { Bilayer } \\
\text { composition } \\
\text { (mol\%) }\end{array}$} & DOTAP & & & & 20 & 40 & 60 \\
\hline & DSPC & 60 & 50 & 40 & 40 & 60 & 40 \\
\hline & POPC & 40 & 50 & 60 & 40 & & \\
\hline $\begin{array}{l}\text { Domain size } \\
\qquad(\mu \mathrm{m})\end{array}$ & & 3 & 2.5 & 2 & 2 & 0.2 & 0.4 \\
\hline
\end{tabular}

\subsubsection{Dependence of bilayer surface charge density on membrane disruption and particle deposition}

The two or three-lipid bilayers were stable in Tris buffer $(0.05 \mathrm{M}, \mathrm{pH} 8.2$,) when the surfaces were scanned upwards and downwards during AFM imaging. The DOTAP may help bilayer formation on negatively charged mica/glass substrates, leading to bilayer formation without any observable pits or cavities. After the addition of $\sim 1 \mathrm{pM}$ of negatively charged AuNPs, four typical changes were plausible: 1) no changes; 2) bilayer was disrupted; 3) NPs were deposited on top of the bilayer; 4) bilayer was disrupted and the NPs were deposited on the disrupted bilayers. These changes can be controlled by the surface charge density of both NPs and the lipid bilayers.

First, we did not observe any changes in AFM imaging and the FL intensity of POPC bilayers after interacting with either fully or partially charged AuNPs. However, in the presence of $\geq 10 \%$ DOTAP, the fully charged AuNPs disrupted the bilayer, leaving large particle aggregates on the mica surface. With partially charged AuNPs, the membrane disruption could be resolved as a function of DOTAP surface concentration (Figure 2.4). At DOTAP concentration below 20\%, random pits or holes were observed after partially charged AuNPs were introduced (Figure 2.4A). The pits were $\sim 5 \mathrm{~nm}$ in depth, corresponding to the bilayer thickness. The disruption seemed to follow the nucleation and growth mechanism. The growth of large cavities could be followed over time using AFM. The pits can grow into a few micrometer cavities within an hour. The AuNPs first peeled off the oppositely charged DOTAP molecules randomly and then peeled off even more at the edges, leading to the pit growth. When the DOTAP concentration was increased to $40 \%$, the bilayer 
was disrupted to the greatest extent (Figure 2.4B), but the disruption process was too fast to be followed by AFM. Increasing the DOTAP concentration further to 60 and $80 \%$ (Figure $2.4 \mathrm{C}, \mathrm{D})$, resulted in a decreased membrane disruption since more and more of the NPs started to deposit at the bilayer surfaces. At 100\% DOTAP concentration, the NPs were densely deposited on the top of bilayers without observable membrane disruption as we have previously shown. ${ }^{24}$

On phase separated DSPC-DOTAP and DSPC-POPC-DOTAP bilayers, the membrane disruption was observed only within the fluidic domains. The gel domains remained intact (Figure 2.5A). The pit sizes were mostly less than $1 \mu \mathrm{m}$. Figure $2.5 \mathrm{~B}$ shows that the membrane was severely disrupted at a concentration of $40 \%$ DOTAP. Figure $2.5 \mathrm{C}$ shows a rather localized AuNPs deposition when DOTAP concentration was increased to $60 \mathrm{~mol} \%$. Most of particles were deposited in a structure resembling the dark striped structures observed at the underlying nanostructured bilayers (Figure 2.2D). The localized particle deposition was due to the localized charge distribution at the bilayer surface. The membrane disruption was negligible in this case and at DOTAP concentration above $60 \%$, indicating that these bilayers are much more stable than the fluidic DOTAP-POPC mixtures (Figure 2.5D).
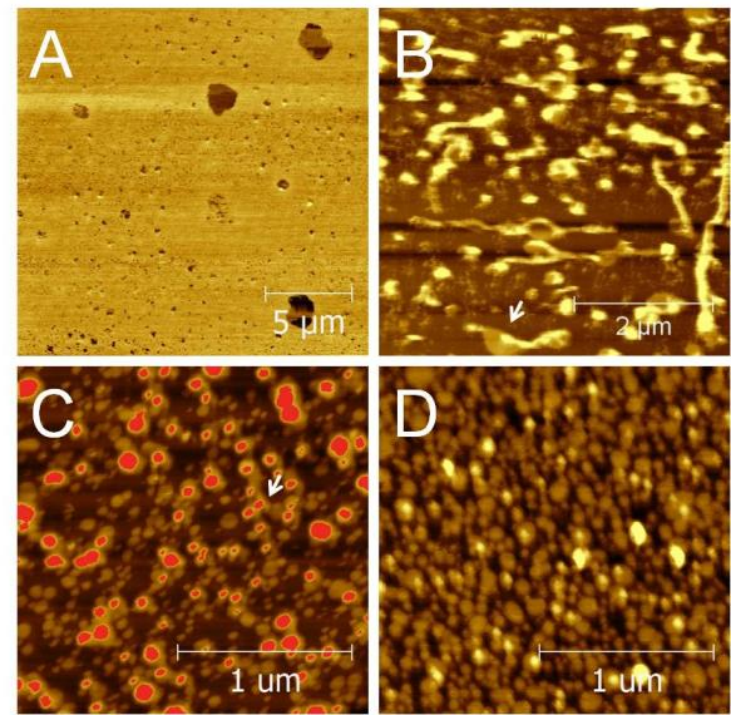

Figure 2.4. AFM images of lipid bilayers after interacting with partially charged AuNPs. (A) 10\% DOTAP-90\%POPC, (B) 40\%DOTAP-60\%POPC, (C) 60\%DOTAP-40\%POPC, (D) 80\%DOTAP-20\%POPC. 

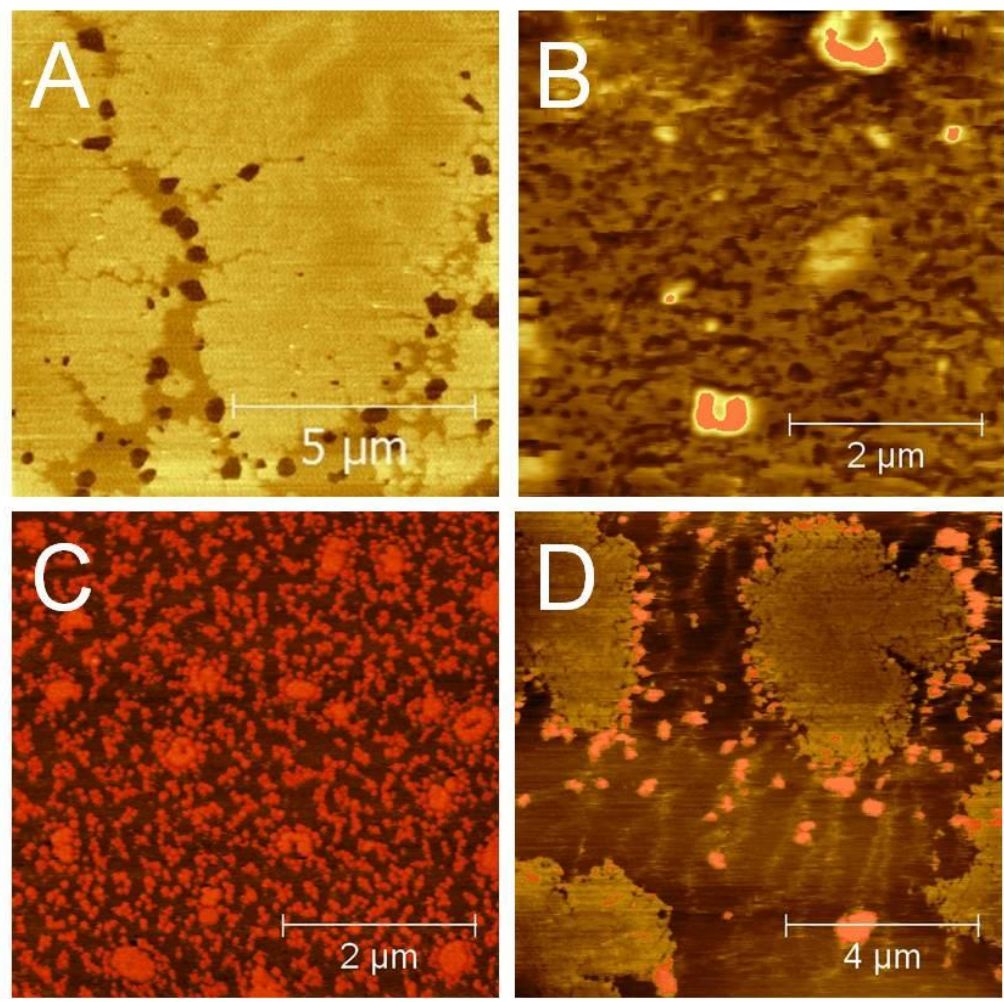

Figure 2.5. AFM images of lipid bilayers after interacting with partially charged AuNPs. (A) $10 \%$ DOTAP-90\%DSPC, (B) 40\%DOTAP-60\%DSPC, (C) 60\%DOTAP-40\%DSPC, (D) 20\%DOTAP-20\%POPC-40\%DSPC.

These experiments strongly suggested that it was the electrostatic attraction that led to membrane disruption. The magnitude of the attraction force and the fluidity of bilayers played important roles in membrane disruption and particle deposition. The dependence of DOTAP concentration on membrane disruption and particle deposition is shown in Figure 2.6. The membranes were greatly disrupted at DOTAP concentrations around $40 \%$. The membrane disruption was likely due to the removal of positively charged DOTAP by the negatively charged AuNPs. Particle deposition was not observed at low DOTAP concentrations, indicating that the local surface did not provide enough attractive force to hold a particle when it was colliding at the surface; but this force was large enough for the particles to remove sufficient DOTAP molecules, leading to pit formation. When the surface charge density was increased, the electrostatic attraction was then large enough to hold NPs, resulting in particle deposition. To confirm that the fluidity does help with membrane 
disruption, we added POPC to the DOTAP-DSPC bilayers. As shown in Figure 2.5D, the fluidic phases were indeed peeled off almost completely, while the gel domains stayed intact.
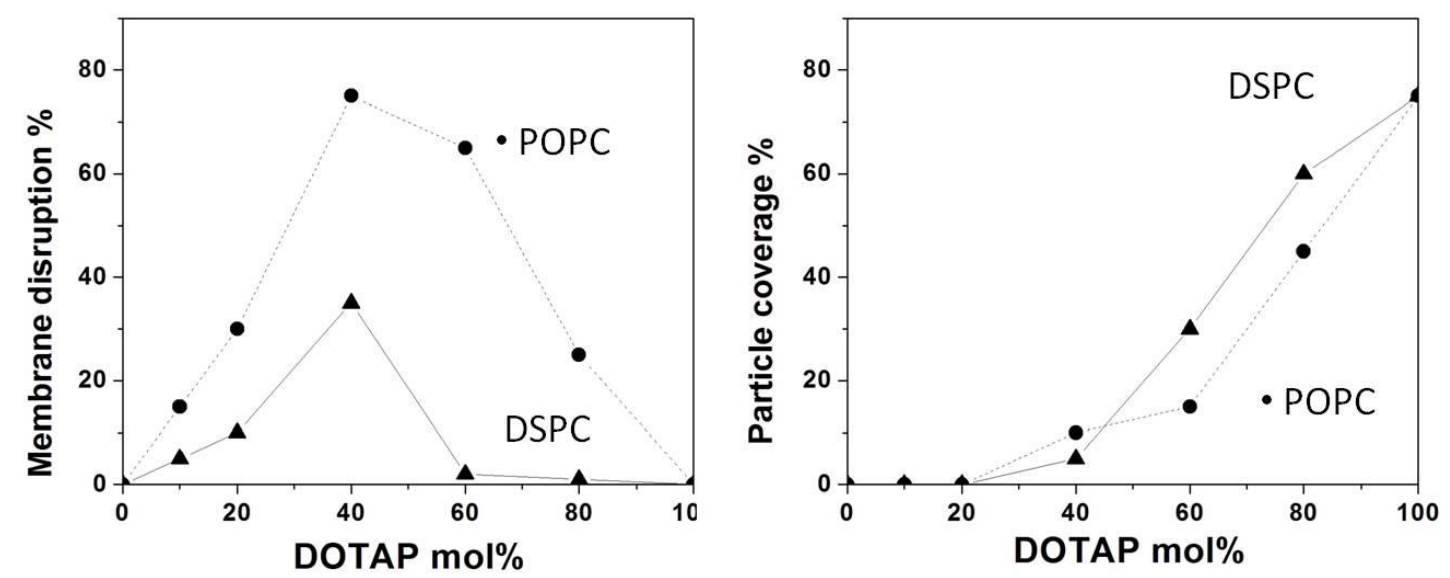

Figure 2.6. Effect of DOTAP concentration on membrane disruption and particle deposition.

\subsubsection{Influence of QDs surface charge on membrane disruption and particle deposition.}

The modulation of the surface charge density at NPs would also provide a means to control the electrostatic interaction with the bilayers. As an example, we used solution $\mathrm{pH}$ to control the surface charge density of $\mathrm{CdSe} / \mathrm{ZnS}$ core/shell QDs. QDs are widely used in biomedical applications as fluorescent tags for drug delivery and bioimaging. ${ }^{25-27}$ The QDs used here were coated with a polymer layer that allowed the dispersion of the QDs in aqueous solutions. The polymer coating has carboxylic acids at the outmost layer. The surface charge density would be tunable by the ratio of neutral $(-\mathrm{COOH})$ and deprotonated anionic $\left(-\mathrm{COO}^{-}\right)$ states of carboxylic acid functional groups. Typical AFM images of the DOTAP bilayer surface are shown in Figure 2.7 after interacting with QDs at different $\mathrm{pH}$ values. At $\mathrm{pH}$ values above 7.2 (Figure 2.7A), the membrane disruption was obvious, and the patched bilayers could be only observed underneath the QDs (Figure 2.7A and inset). The QDs were surprisingly aggregated as two-dimensional stripes, possibly due to the bilayer fluidity. When the $\mathrm{pH}$ value was decreased to 6.5 and 5.5, more and more QDs were aggregated as twodimensional particle arrays. Further decreasing the $\mathrm{pH}$ to 5.0 and below 5.0 led to deposition of individual QDs densely packed at the bilayer surfaces. Since the charges carried by DOTAP molecules were independent of $\mathrm{pH}$ value, the decrease in $\mathrm{pH}$ value would only decrease the amount of negative charges or charge density at the QDs surface. The highly 
charged QDs at higher $\mathrm{pH}$ values can easily disrupt the charged bilayers, similarly to the fully charged AuNPs as shown in Figure 2.8.
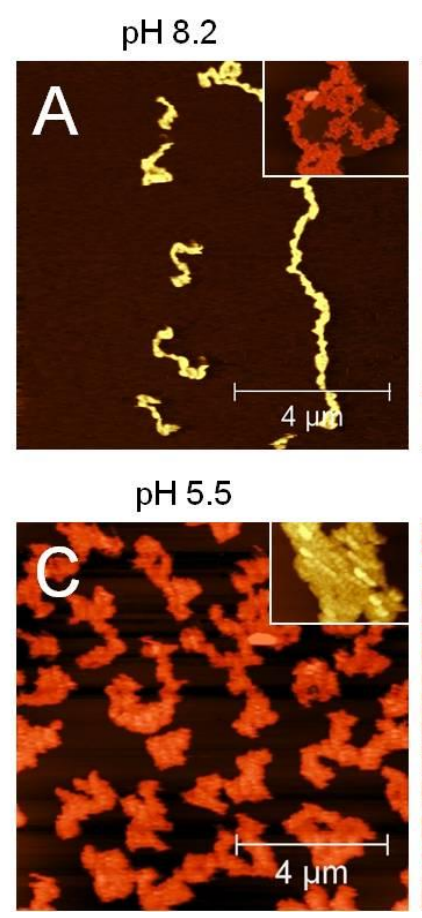

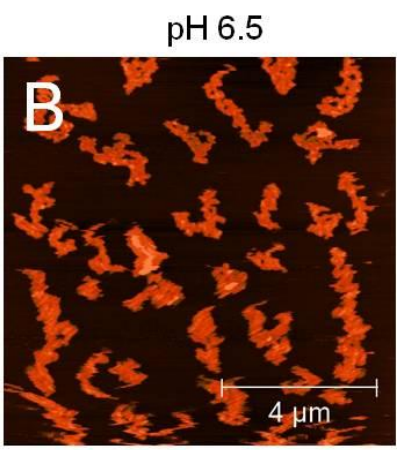

$\mathrm{pH} 5.0$

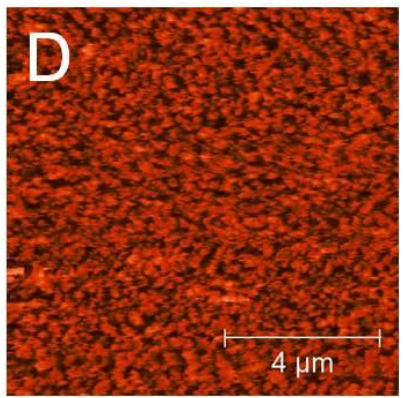

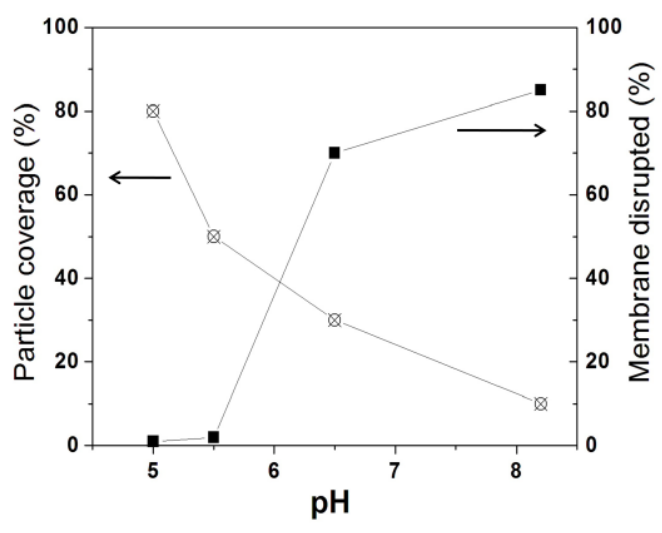

(Left) Figure 2.7. AFM images of DOTAP lipid bilayers after interacting with quantum dots at different solution pHs.

(Right) Figure 2.8. Dependence of $\mathrm{pH}$ on membrane disruption and deposition of carboxylic acid terminated QDs at DOTAP bilayers.

\subsection{Conclusions}

We have varied the surface charge density of both NPs and supported lipid bilayers, and monitored the consequence of their interactions by atomic force microscope and fluorescent microscope. Depending on their surface charge densities, negatively charged AuNPs can deposit at the membrane surfaces or extract the oppositely charged DOTAP molecules to disrupt the membranes. The membrane disruption selectively occurred in the fluidic domains. The particle deposition was uniform over the surfaces of DOTAP and DOTAP-POPC bilayers, an indication of homogeneous charge distribution, while at nanostructured DOTAPDSPC surfaces, the AuNPs were deposited into the network structures. This work demonstrated that the surface charge localization could lead to localized membrane disruption and particle deposition. By controlling the surface charge density of both NPs and 
lipid bilayers, we can modulate their electrostatic interactions that would result in welldefined membrane disruption and NP surface assembly. Better understanding and modeling the electrostatic interaction forces would enable us to control many important physiological events, including nanomaterials uptake or release.

\subsection{References}

1) Kelly, C. V.; Leroueil, P. R.; Orr, B. G.; Banaszak Holl, M. M.; Andricioaei, I. J Phys Chem B. 2008, 112, 9346-9353.

2) Goodman, C. M.; McCusker, C. D.; Yilmaz, T.; Rotello, V. M. Bioconjugate Chem. 2004, 15, 897-900.

3) Mecke, A.; Lee, D. K.; Ramamoorthy, A.; Orr, B. G.; Banaszak Holl, M. M. Langmuir, 2005, 21, 8588-8590.

4) Mecke, A.; Majoros, I. J.; Patri, A. K.; Baker, J. R.; Banaszak Holl, M. M.; Orr, B. G. Langmuir 2005, 21, 10348-10354.

5) Chen, J. M.; Hessler, J. A.; Putchakayala, K.; Panama, B. K.; Khan, D. P.; Hong, S.; Mullen, D. G.; DiMaggio, S. C.; Som, A.; Tew, G. N.; Lopatin, A. N.; Baker, J. R.; Holl, M. M. B.; Orr, B. G. J. Phys. Chem.B 2009, 113, 11179-11185.

6) Arvizo, R.R.; Miranda, O. R.; Thompson, M. A.; Pabelick, C. M.; Bhattacharya, R.; Robertson, J. D.; Rotello,V. M.; Prakash, Y. S.; and Mukherjee, P. Nano Lett. 2010, 10, 2543-2548.

7) Goodman, C. M.; McCusker, C. D.; Yilmaz, T.; Rotello, V. M. Bioconjugate Chem. 2004, 15, 897-900.

8) Lin, J.; Zhang, H.; Chen, Z.; Zheng Y.; ACS Nano

9) Hong, S.; Leroueil, P. R.; Janus, E. K.; Peters, J. L.; Kober, M. M.; lslam, M. T.; Orr, B. G.; Baker, J. R., Jr.; Banaszak Holl, M. M. Bioconjugate Chem.2006, 17, 728-734.

10) Leroueil, P. R.; Hong, S.; Mecke, A.; Baker, J. R., Jr.; Orr, B. G.; Banaszak Holl, M. M. Acc. Chem. Res. 2007, 40, 335-342.

11) Leroueil, P. R.; Berry, S. A.; Duthie, K.; Han, G.; Rotello, V. M.; McNerny, D. Q.; Baker, J. R., Jr.; Orr, B. G.; Banaszak Holl, M. M. Nano Lett. 2008, 8, 420-424.

12) Antonov, V. F.; Petrov, V. V.; Molnar, A. A.; Predvoditelev, D. A.; Ivanov, A. S.; Nature, 1980, 283, 585-586. 
13) Ginzburg, V. V.; Balijepailli, S.; Nano Lett. 2007, 7, 3716-3722.

14) Liu, J.; Groves, J. T.; Chakraborty, A. K. J. Phys. Chem. B 2006, 110, 8416-8421.

15) Brust, M.; Walker, M.; Bethell, D.; Schiffrin, D. J.; Whyman, R. J. Chem. Soc., Chem. Commun. 1994, 801.

16) Smetana, A. B.; Wang, J. S.; Boeckl, J.; Brown, G. J.; Wai, C. M. Langmuir 2007, 23, 20429.

17) Liu, J.; Qi, S.; Groves, J. T.; Chakraborty, A. K. J. Phys. Chem. B 2005, 109,1996019969.

18) Spurlin, A. A.; Gewirth A. A. J. Am. Chem. Soc. 2007, 129, 11906-11907.

19) Lin W. C, Blanchette C. D, Ratto T. V, Longo M. L. Methods Mol Biol. 2007, 400, 50313.

20) Lin, W. C, Blanchette, C. D, Ratto, T. V, Longo, M. L. Biophys J. 2006, 90, 228-237.

21) Rinia, H. A, de Kruijff, B. FEBS Lett. 2001, 504, 194-199.

22) Bernchou, U.; Ipsen, J. H.; Simonsen, A. C. J. Phys. Chem. B 2009, 113, 7170-7177.

23) Nussio, M. R.; Lowe, R. D.; Voelcker, N. H.; Flavel, B. S.; Gibson, C. T.; Sykes, M. J.; Miners, J. O.; Shapter, J. G. SOFT MAT 2010, 6, 2193-2199.

24) Xiao, X. Y.; Montaño, L. G.; Allen, A.; Achyuthan, E.K.; Wheeler, R. D.; Brozik S. M.; Langmuir, 2011, 27, 9484-9489.

25) Alivisatos, P. Nat. Biotechnol. 2004, 22, 47-52.

26) Michalet, X., Pinaud, F. F., Bentolila, L. A., Tsay, J. M., Doose, S., Li, J. J., Sundaresan, G., Wu, A. M., Gambhir, S. S., and Weiss, S. Science, 2005, 307, 538-544.

27) Medintz, I. L., Uyeda, H. T., Goldman, E. R., and Mattoussi, H. Nat. Mater. 2005, 4, 435-446. 


\section{Lipid Bilayer Templated Gold Nanoparticles Nanoring Formation Using ZIRCONIUM ION COORDINATION CHEMISTRY}

\subsection{Introduction}

Lipid bilayer assemblies (LBAs) on solid substrates are widely used to mimic the dynamics and structural features of cell membranes. ${ }^{1,2}$ Using LBAs, it is possible to mimic and investigate biologically relevant processes such as membrane protein recognition and channel function as well as use the LBAs for biomaterials or biomedical applications. For example, biosensors can be created by the incorporation of ion channel proteins as well as other membrane protein receptors. ${ }^{3-6}$ The formation of LBAs on solid supports typically occurs with a 10 to $20 \AA$ thick hydration layer between the bilayer and the support (substratum) affording the LBAs lateral fluidity. ${ }^{7,8}$ Accordingly, while supported, the LBAs are typically able to maintain their intrinsic structural and functional properties. This is illustrated by examples of lipids such as palmitoyl oleoyl phosphatidyl choline (POPC) and N-[1-(2,3dioleoyloxy)propyl]-N,N,N-trimethylammonium methylsulfate (DOTAP) with solid phase transition temperatures below room temperature that result in fluid LBAs at room temperature, whereas saturated lipids such as distearylphosphatidyl choline (DSPC) result in non-fluid LBAs at room temperature. Likewise, a combination of two or more lipid mixtures can result in two-dimensional phase separation of the various lipid components. ${ }^{9,10}$ Although the supported bilayers can be assembled upon a variety of solid surfaces by vesicle fusion and Langmuir-Blodgett techniques, their detailed structures are often complex and strongly related to the lipid composition, the nature of the surface and assembly conditions such as temperature, ionic strength and $\mathrm{pH} .{ }^{11}$

Depending on the environmental conditions used, phenomena such as bilayer curvature and formation of nanodomains and nanopores have been observed. ${ }^{12-17}$ Other work has focused upon the interactions of synthetic organic and inorganic nanoparticles (NPs) with biological membranes. ${ }^{18-21}$ Here we report the organization of carboxylate terminated gold nanoparticles (AuNPs) that interact with a positively charged DOTAP lipid bilayer membrane. The AuNPs are shown to organize into nanoring architectures due to a combination of zirconium 
coordination chemistry $^{22}$ and secondary acidic disruption of the LBA due to addition of $\mathrm{ZrCl}_{4}$. Potential applications of these nanoring structures are briefly discussed.

\subsection{Materials and Methods}

AuNPs Synthesis. AuNPs were prepared using published methods with minor modifications $^{23-24}$. 1-dodecanethiol coated AuNPs (i.e., AuNP-DDT) were prepared using a two-phase liquid-liquid reaction system. The fully carboxylated AuNPs (fc-AuNPs), such as 11-mercaptoundecanoic anion and 3-mercaptopropionic acid coated AuNPs (i.e., Au-MUA and Au-MPA, respectively), were prepared by the inverse microelmusion method. The partially carboxylated AuNPs (pc-AuNPs) were obtained after a ligand exchange reaction between DTT and MUA.

Lipids and Liposomes. Lipids, DOTAP, DSPC and POPC were purchased from Avanti Polar Lipids and stored in chloroform. Liposomes were prepared by probe sonication. $20 \mathrm{mg}$ lipid powder was dissolved in $1 \mathrm{ml}$ chloroform. $76 \mu \mathrm{l}$ of such solution was dried under nitrogen stream and under house-vacuum for more than $2 \mathrm{~h}$. The lipid cake was then hydrated with N-2-hydroxyethylpiperazine-N'2-ethane sulfonate (HEPES) buffer (150 mM NaCl +20 $\mathrm{mM} \mathrm{CaCl} 2+10 \mathrm{mM}$ HEPES, $\mathrm{pH}$ 7.4). After 5 freeze-thaw cycles the solution was probe sonicated for $10 \mathrm{~min}$. The size of the liposomes was fairly monodisperse around 30 to $40 \mathrm{~nm}$.

Lipid Bilayer Assembly. Supported lipid bilayers were assembled by placing liposome solutions on freshly cleaved mica or piranha (75 vol\% sulfuric acid) cleaned glass coverslips. The bilayers were first rinsed with water and then Tris- $\mathrm{HCl}$ buffer (50 mM Tris, $\mathrm{pH} 8.2$ ).

AuNPs Binding. Fully and partially carboxylated AuNPs were dissolved in Tris buffer, pH 8.2, and were directly introduced onto the supported lipid bilayers by electrostatic interaction. Above $1 \mathrm{nM}$ of particle concentration, the particle coverage reached saturation.

HCl or ZrCl4 Treatment. After binding AuNPs, usually for 10-30 min after introducing particle solutions, the surfaces were then rinsed with Tris buffer and water to remove unbound particles. The solution was then exchanged by $0.01-0.1 \mathrm{MHCl}$ or $0.005-0.1 \mathrm{MZrCl} 4$ twice. In AFM experiments, the acidic solutions were then exchanged to DI-water followed 
by Tris buffer for imaging. In fluorescence experiments, the samples were directly imaged in the presence of $\mathrm{HCl}$ or $\mathrm{ZrCl}_{4}$.

Atomic Force Microscopy (AFM). We used Agilent Pico SPM II and MAC Type II cantilevers with a typical spring constant of $\sim 1 \mathrm{~N} / \mathrm{m}$. All images were taken in a liquid cell in MAC mode (set point, $\sim 75 \%$ of free oscillation, scan rate, $\sim 1 \mathrm{~Hz}$ ). To mechanically scratch the particle-lipid bilayer surfaces, the set point was further decreased to $\sim 30 \%$ of the free oscillation. The image analysis was performed using Gwyddion software.

Fluorescence Microscopy (FL). We used an Olympus 1X-71 inverted optical microscope to generate a TIF file of the fluorescence image and then analyzed using Gwyddion software for the scale bar, color and color scale, and optical dimensions. 0.3\% of $\beta$-BODIPY ${ }^{\circledR}$ FL C $_{12^{-}}$ HPC (Invitrogen, Life Technologies, Carlsbad, CA) in lipid bilayers was used. The dye had an excitation and emission maxima of 506 and 513nm, respectively.

\subsection{Results and discussion}

\subsubsection{AuNPs Assembly}

The core diameter of both fc-AuNPs and pc-AuNPs was 3 to $5 \mathrm{~nm}$ and their hydrodynamic size around $10 \mathrm{~nm}$ as determined by TEM and DLS, respectively. The absorption peak of the pc-AuNPs was at $\sim 512 \mathrm{~nm}$. AuNPs coated by a pure monolayer of MUA or MPA had an absorption maximum of $\sim 532 \mathrm{~nm}$ (Figure 3.1). The particles were soluble at $\mathrm{pH}$ greater than 7.0 and were stable for several months. 

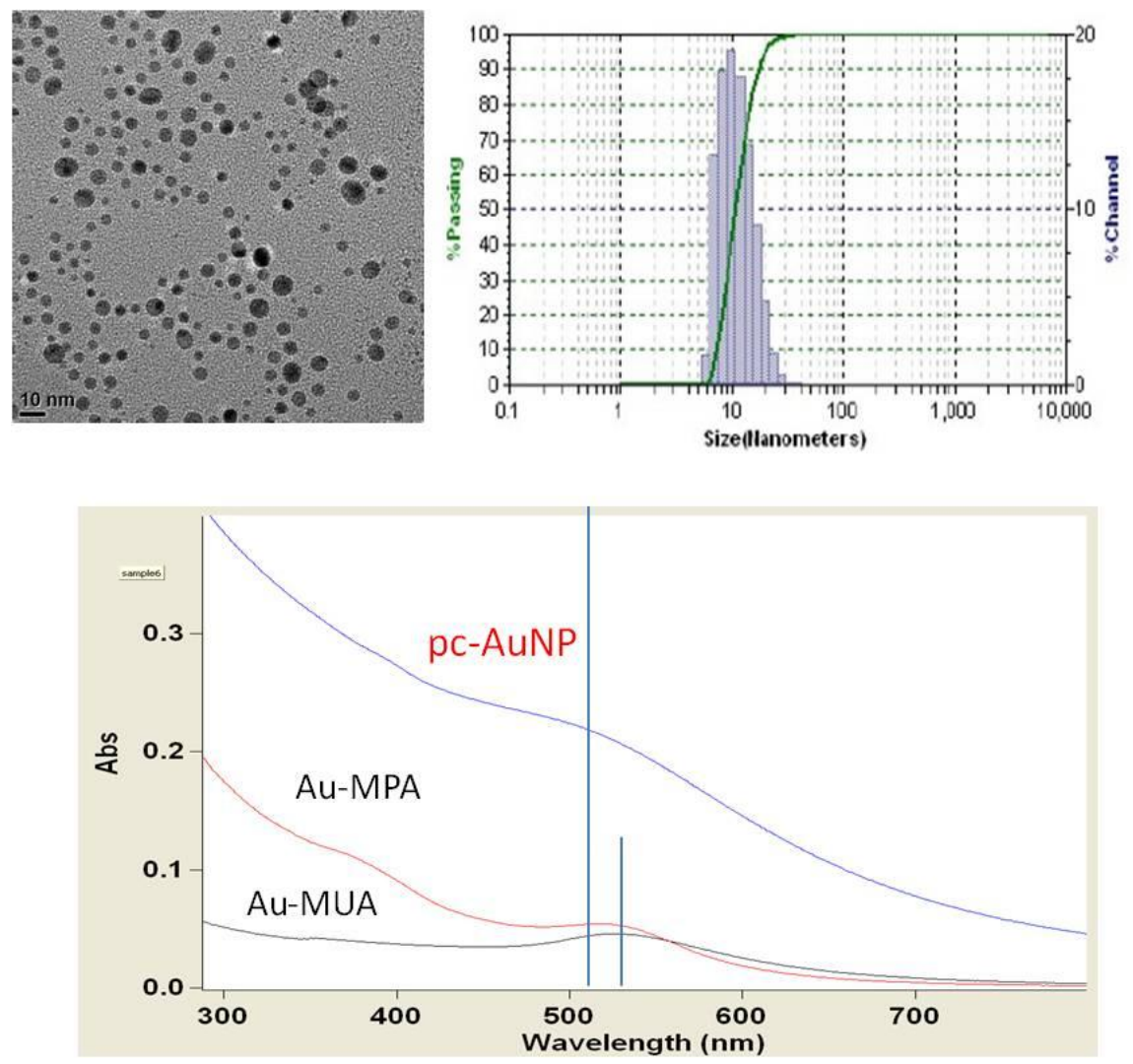

Figure 3.1. TEM, DLS and UV-Vis spectrum of pc-AuNPs. The UV-Vis spectra of Au-MUA and Au-MPA were included for comparison.

Initially, we attempted to assemble the AuNPs onto DOTAP LBAs using fully carboxylated AuNPs, Au-MUA or Au-MPA, based on the electrostatic attraction between the oppositely charged lipid head group and the AuNPs. However, we found that these negatively charged AuNPs often disrupted or peeled off the positively charged DOTAP membranes, similar to the observations made by others. ${ }^{25,26}$ We therefore synthesized partially carboxylated AuNPs with much less surface charge and assembled these particles on the LBA. Figure 3.2 illustrates the proposed molar ratio between MUA and DDT molecules adsorbed at the AuNP surfaces. DOTAP is a cationic lipid with a net positively charged head group. Figure 3.3A shows a typical AFM image of a DOTAP bilayer on mica before pc-AuNPs were assembled. The image is almost featureless since the DOTAP bilayer remains fluidic at room temperature. Figure 3.3B shows the DOTAP LBA after addition of 1nM pc-AuNP solution at $\mathrm{pH} \sim 8$. The spherical nanoparticles of rather uniform size distribution are observed on the 
LBA surface with no disruption of the underlying LBA. We found that pc-AuNPs did not assemble on the POPC bilayers presumably due to a net zero surface charge. The surface coverage of AuNPs was also shown to be controllable by varying particle concentrations or replacing the DOTAP partially by POPC and DSPC which are zwitterionic lipids with zero net surface charges.
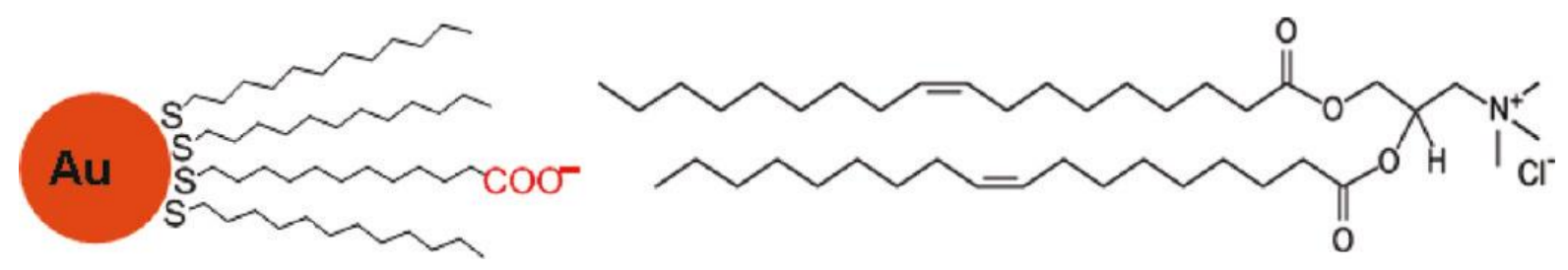

Figure 3.2 Schematics of negatively charged AuNP and positively charged DOTAP lipid.

The electrostatic interactions between DOTAP bilayers and pc-AuNPs were investigated by AFM. Figure 3.3B is a typical surface of pc-AuNPs assembled on the DOTAP bilayer. After an initial scan of such a surface area under a rather large force, i.e., scanning the surface at an amplitude setpoint $\sim 30 \%$ of the free oscillating amplitude, a second, larger area scan at normal $75 \%$ of the free oscillating amplitude was taken (Figure 3.3C). As can be observed, most of the pc-AuNPs have been pushed away by the AFM tip during the initial scan leaving a sparsely covered DOTAP LBA in the area of the previous scan. The LBA appears to remain intact upon both pc-AuNPs deposition and forced movement within the detection limit of AFM. Figure 3.3D shows that the particles are about $5 \mathrm{~nm}$ above the bilayer, which is close to the hydrodynamic radius of pc-AuNPs measured by DSL. 

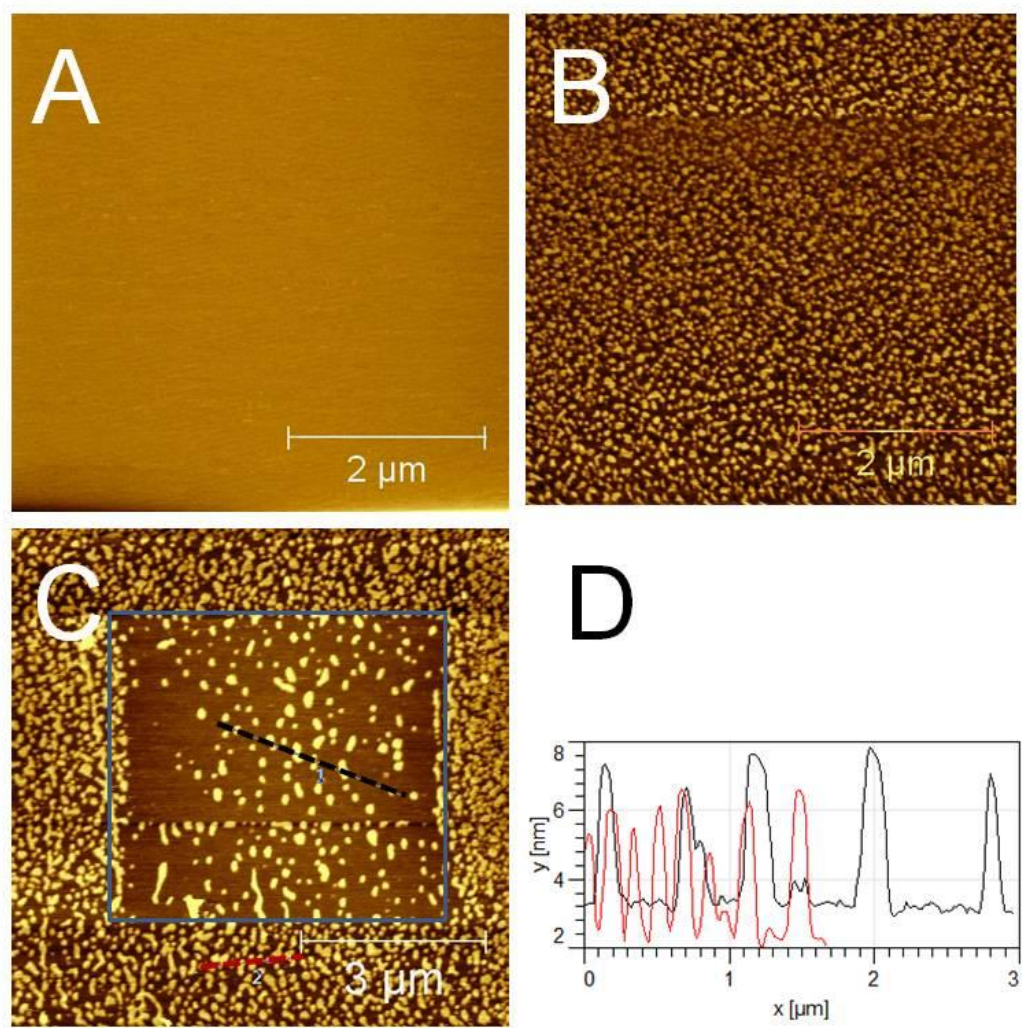

Figure 3.3. AFM images of DOTAP bilayer before (A) and after (B) the addition of pcAuNPs ( 1nM). (C) AFM image showing pc-AuNPs being pushed away from the center square by an AFM tip and (D) the height analysis along the lines shown in (C).

\subsubsection{ZR ION INDUCED NANORING FORMATION.}

$\mathrm{ZrCl}_{4}$ has the potential to coordinate with the carboxylic groups on the functionalized AuNPs.

Figure 3.4A shows that $\sim 250 \mathrm{~nm}$ inner diameter sized nanorings were formed immediately after $5 \mathrm{mM} \mathrm{ZrCl}_{4}$ solution was added. Figure 3.4B and the corresponding phase image (not shown) indicate that the underlying bilayer appeared to be unaltered. The inside of the nanorings is as flat as previous fluidic bilayer surfaces. The AFM height analysis still shows 6 to $10 \mathrm{~nm}$ in height difference corresponding to the radius of AuNPs (Figure 3.4C). In addition to nanoring formation, the rest of the bilayer was covered by individual particles or relatively larger islands, an indication of short-distance limited reorganization of AuNPs. 

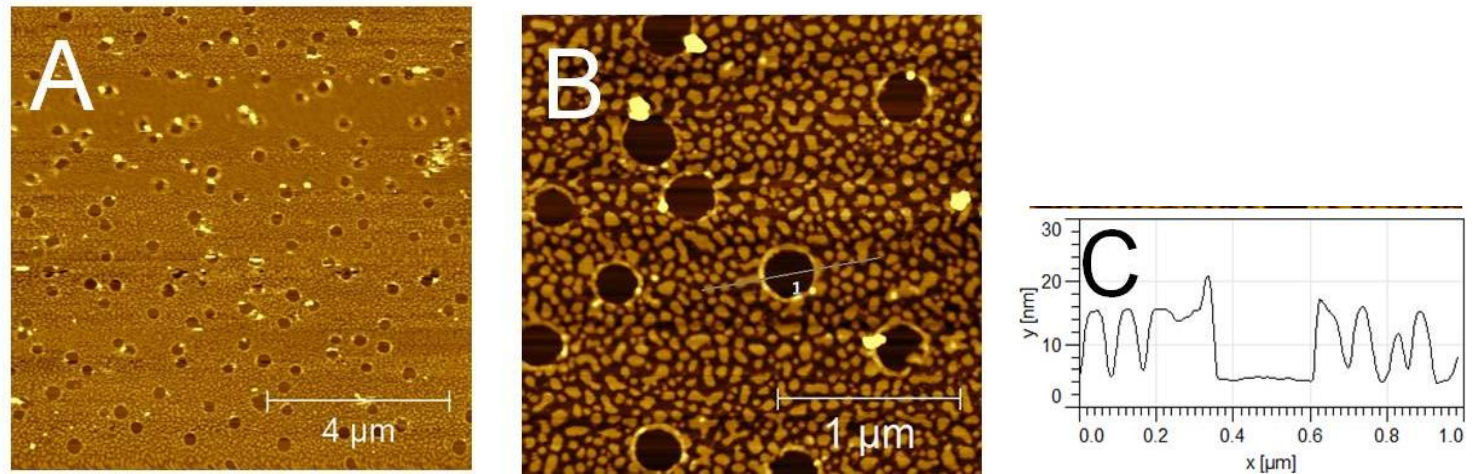

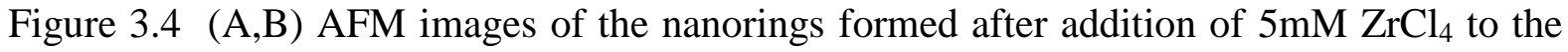
DOTAP bilayer covered with pc-AuNPs. (C) Height analysis along the line shown in (B).

We have examined and found several experimental conditions that affected the formation of nanorings. First, the diameter of nanorings was decreased when a higher concentration of $\mathrm{ZrCl}_{4}$ was used. Figure 3.5 shows the size distribution of the nanorings analyzed from 3 to 4 surface locations for three $\mathrm{Zr}$ ion concentrations. The ring sizes were inversely related to $\mathrm{Zr}$ ion concentration. Second, the addition of $\mathrm{ZrCl}_{4}$ in the presence of acetic acid, i.e., $\mathrm{Zr}$ ions were fully coordinated with acetate, did not lead to any ring formation (not shown), suggesting that free $\mathrm{Zr}$ ions were needed to coordinate with carboxylic groups among the AuNPs. Third, the addition of $\mathrm{Cu}$ or $\mathrm{Ni}$ ions, instead of $\mathrm{Zr}$ ions, did not result in nanoring formation. Fourth, nanorings were not formed when DOTAP was partially replaced by DSPC at more than $20 \%$, indicating that bilayer fluidity plays a role in ring formation. Finally, when the $\mathrm{Zr}$ ion solutions were carefully buffered at $\mathrm{pH}$ above 3, no nanorings were observed. The dependence of ring formation on $\mathrm{pH}$ indicates that the coordination of $\mathrm{Zr}$ ions with carboxylic groups alone is not able to induce AuNP reorganization at $\mathrm{pH}$ above 3 . The reorganization of AuNPs requires the particles to move above the bilayers, which is possibly hindered by the weakly electrostatic attraction from the lipid head groups. 

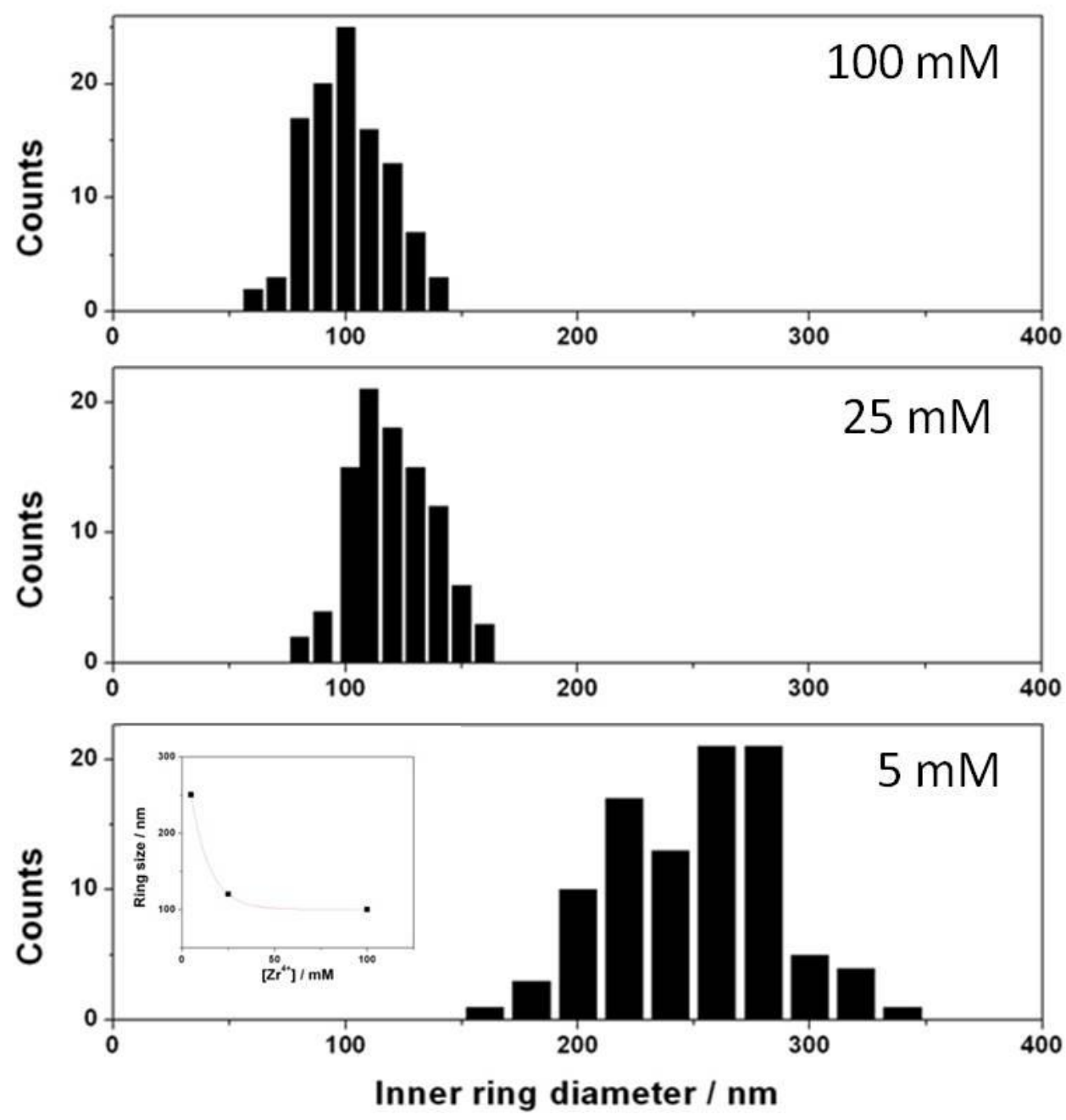

Figure 3.5 Dependence of nanoring size distribution on zirconium ion concentration.

\subsubsection{ACID-INDUCED BILAYER DISRUPTION AND BUDDING.}

The $\mathrm{pH}$ values of the $\mathrm{ZrCl}_{4}$ solutions for the three concentrations were: $2.06(5 \mathrm{mM}), 1.68$ $(25 \mathrm{mM})$ and $1.36(100 \mathrm{mM})$. To determine the $\mathrm{pH}$ effect in the process of AuNP reorganization, we treated the LBA-pc-AuNPs surface with $0.01 \mathrm{M} \mathrm{HCl}, \sim \mathrm{pH} 2.0$, in the place of unbuffered $\mathrm{ZrCl}_{4}$ solution. As can be seen from Figure3.6B, $\mathrm{HCl}$ addition resulted in extensive membrane disruption. The residual disc-like patches were possibly covered by AuNP aggregates, leading to the height variations greater than the sum of the bilayer thickness $(\sim 6 \mathrm{~nm})$ and the AuNP radius (Figure 3.6C). In contrast to $\mathrm{ZrCl}_{4}$ treated LBAs, no ordered ring-structures were formed. 

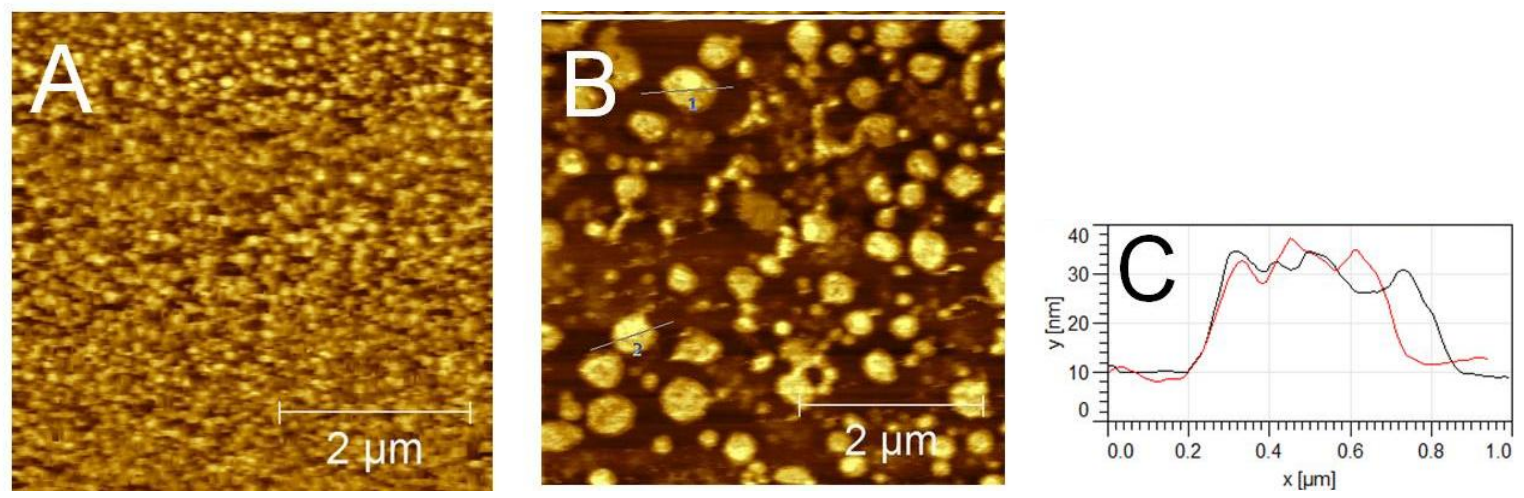

Figure 3.6. AFM images of the DOTAP bilayer covered with pc-AuNPs before (A) and after (B) the addition of $10 \mathrm{mM} \mathrm{HCl}$. (C) Height analysis along the lines shown in (B).

We also investigated the effect of the addition of $\mathrm{ZrCl}_{4}$ on $\mathrm{LBAs}$ alone. Figure 3.7 shows a typical fluorescence microscopy image of a DOTAP bilayer on glass before and after the addition of $5 \mathrm{mM} \mathrm{ZrCl}_{4}$. The addition of $\mathrm{ZrCl}_{4}(\mathrm{pH} 2.06)$ disrupted the DOTAP LBA in two ways, creating large patches in the LBA as well as resulting in the appearance of circular hole like structures of rather uniform sizes (Figure 3.7B). These hole-like structures were confirmed to be three dimensional (3D) bubbles by adjusting the $Z$ plane of the microscope focus. These 3D bilayer structures are similar to earlier structures created by Hovis et al. and recently by Goertz et al. ${ }^{27-29}$ The later work showed very similar 3D structures in POPC bilayers at the extreme low and high pHs. In our case, the formation of 3D bubbles is likely due to the protonation of the glass substratum, which altered the stability of bilayers interacting with the substrates.

To determine the effect of AuNPs on membrane disruption, we further used fluorescence microscopy to look at DOTAP LBAs covered with pc-AuNPs and followed by the same $\mathrm{ZrCl}_{4}$ treatment. Figure 3.8 shows the bright nanorings above the lipid bilayers and large area bilayer disruptions as well. Optical examination demonstrated that the ring diameters were $300 \mathrm{~nm}$ which although at the optical detection limit, were nevertheless in agreement with our AFM data (Figure 3.4), suggesting that they are indeed nanorings of pc-AuNPs. Based upon these data, we concluded that unlike the bubbles observed in Figures 3.7B for bilayers treated with $\mathrm{ZrCl}_{4}$ in the absence of pc-AuNPs, the bright nanorings of pc-AuNPs on DOTAP represented zirconium-coordinated gold nano-structures. The brightness of nanorings in 
Figure 3.8 is presumed to be due to local surface plasmon resonance between the pc-AuNPs and the $\beta$-BODIPY dye molecules, ${ }^{30,31}$ since the absorption maximum of the pc-AuNPs (512 $\mathrm{nm})$ overlapped with the excitation maximum $(506 \mathrm{~nm})$ of the dye molecules. The nanorings have higher density or closer package of AuNPs and thus more enhanced fluorescent emissions.
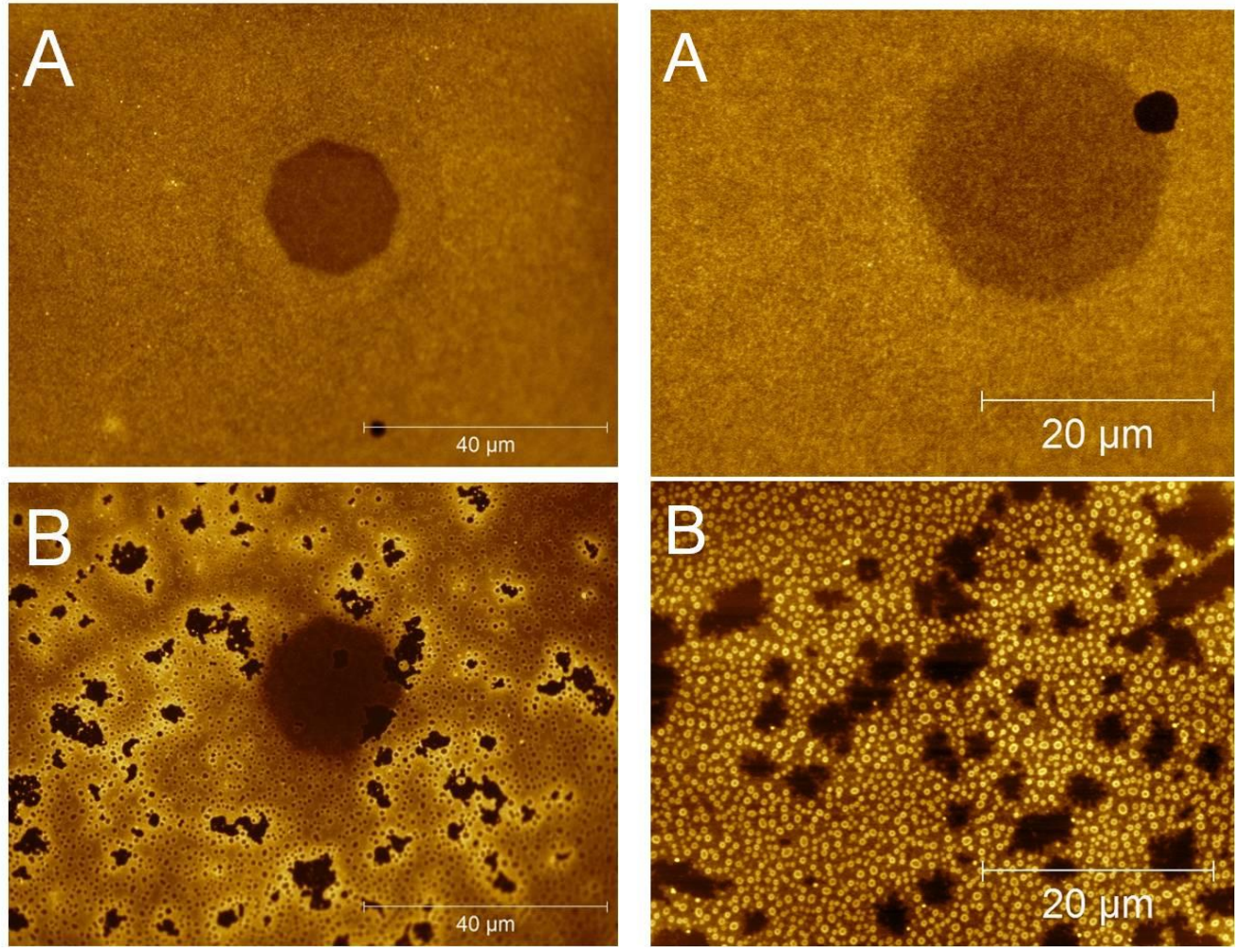

(Left) Figure 3.7 Fluorescence images of a DOTAP bilayer on glass before (A) and after (B) the addition of $5 \mathrm{mM} \mathrm{ZrCl}_{4}$ in the absence of AuNPs.

(Right) Figure 3.8. Fluorescence images of DOTAP bilayer covered with pc-AuNPs before (A) and after (B) the addition of $5 \mathrm{mM} \mathrm{ZrCl}_{4}$.

\subsubsection{PROPOSED Ring Formation MECHANISM.}

The formation of zicornate $\left(\mathrm{ZrO}_{2}\right)$ rings was excluded in our acidic test solutions. When dissolved in water, $\mathrm{ZrCl}_{4}$ is readily hydrolyzed to form a variety of derivatives of the zirconyl, dizirconyl and similar cationic radicals depending on the acidic environment. ${ }^{32,33}$ In general, $\mathrm{Zr}(\mathrm{OH})_{4}$ or $\mathrm{ZrO}_{2}$ is formed only at $\mathrm{pH}$ above 4 , at which $\mathrm{pH}$ values we did not observe the formation of nanorings. At lower $\mathrm{pH}$ values, the cationic zirconyl monomers $(\mathrm{pH}<1)$ and polymers $(\mathrm{pH} \leq 4)$ still strongly coordinate with carboxylic acid by breaking the 
zirconyl-oxygen-zirconyl bridges in their polymeric complexes. ${ }^{34,35}$ In our study $(\mathrm{pH}<3)$, each zirconyl polymeric radical was likely capable of coordinating more than two carboxylic acid termini from the same or neighboring AuNPs, leading to the reorganization of AuNPs in the course of an acid-induced membrane budding event.

$$
\begin{aligned}
& \mathrm{ZrCl}_{4} \text { hydrolysis at } \mathrm{pH}<3: \mathrm{Zr}^{4+}+\mathrm{xH}_{2} \mathrm{O} \longrightarrow \mathrm{Zr}(\mathrm{OH})_{\mathrm{x}}{ }^{4-\mathrm{x}}+\mathrm{xH}^{+} \\
& \mathrm{Zr} \text { ion coordination: mAuNP-COOH }+\mathrm{Zr}(\mathrm{OH})_{\mathrm{x}}{ }^{4-\mathrm{x}} \longrightarrow \mathrm{Zr}(-\mathrm{OOC}-\mathrm{AuNP})_{\mathrm{m}}+\mathrm{nH}_{2} \mathrm{O}
\end{aligned}
$$

Zirconium coordination is required in the process of nanoring formation. Such coordination may lead to enhance the stability of AuNPs above bilayers by holding them together and the stability of the bilayers against acid-induced disruption as well. When the $\mathrm{pH}$ decreases in the presence of zirconium coordinated AuNPs, the vesicle budding could only occur in the spaces between the coordinated AuNPs. The net result was the observed nanorings. This mechanism also explains the inverse correlation between the nanoring diameter and the $\mathrm{ZrCl}_{4}$ concentration. Increased $\mathrm{ZrCl}_{4}$ concentrations resulted in enhanced AuNP coordination leading to greater stability. Thus, only small vesicles were allowed to form and bud off from the LBA surface. It is not fully understood why both AFM and fluorescence images showed the intact bilayers inside the nanorings. However, it is possible that the bilayer budding was initiated by the acid but eventually did not take place or was suppressed by the nanorings. It is also possible that the nanopores within the DOTAP lipid bilayer are back-filled through the bilayer lateral diffusion or from the bulk solution. Addition of $\mathrm{Cu}$ or $\mathrm{Ni}$ ions did not result in nanoring formation since their complexes with carboxylic group were not stable in low $\mathrm{pH}$ solutions.

\subsection{Conclusions}

We have demonstrated that a combination of the unique properties of DOTAP LBAs and zirconium coordination chemistry of partially carboxylated AuNPs resulted in the formation of gold nanoring nanostructures. These structures rely on the native lateral fluidity of the LBAs, the $\mathrm{pH}$ response of the $\mathrm{LBAs}$ to the $\mathrm{ZrCl}_{4}$ and zirconium coordination of the AuNPs. Our approach to gold nanoring formation utilized coordination chemistry and is therefore a much simpler technique compared to colloidal or nanoimprint lithography for gold nanoring 
fabrication. ${ }^{36}$ Our data also suggests potential attributes for materials that could be used for future design and programming of biohybrid nanomaterial composites with interesting sensor properties. ${ }^{13}$ For example, gold nanorings in a size regimen $(75$ to $150 \mathrm{~nm}$ ) similar to those described here were used to detect protein-ligand interactions. ${ }^{36}$ Other potential applications include nanorings functioning as storage cavities for proteins or drugs and as near-infrared surface-enhanced spectroscopic sensors. ${ }^{37,38}$ Our future studies will explore these applications.

\subsection{References}

1) Sackmann, E. Science 1996, 271, 43.

2) Ranganathan, R. Science 2007, 318, 1253.

3) Cornell, B. A.; Braach-Maksvytis, V. L. B.; King, L. G.; Osman, P. D. J.; Raguse, B.; Wieczorek, L.; Pace, R. J. Nature 1997, 387, 580.

4) Menger, F. M.; Galloway, A. L.; Chlebowski, M. E.; Wu, S. J. Am. Chem. Soc. 2006, 128, 14034.

5) Hayden, C. C.; Hwang, J. S.; Abate, E. A.; Kent, M. S.; Sasaki, D. Y. J. Am. Chem. Soc. 2009, 131, 8728.

6) Jonsson, M. P.; Jonsson, P.; Dahlin, A. B.; Hook, F. Nano Lett 2007, 7, 3462.

7) Zwang, T. J.; Fletcher, W. R.; Lane, T. J.; Johal, M. S. Langmuir 2010, 26, 4598.

8) Hartshorn, C. M.; Jewett, C. M.; Brozik, J. A. Langmuir 2010, 26, 2609.

9) Spurlin, T. A.; Gewirth, A. A. J. Am. Chem. Soc. 2007, 129, 11906.

10) Zhang, L. F.; Granick, S. MRS Bulletin 2006, 31, 527.

11) Castellana, E. T.; Cremer, P. S. Surf. Sci. Rep. 2006, 61, 429.

12) Roiter, Y.; Ornatska, M.; Rammohan, A. R.; Balakrishnan, J.; Heine, D. R.; Minko, S. Nano Lett 2008, 8, 941.

13) Tribet, C.; Vial, F. Soft Matter 2008, 4, 68.

14) Denisov, I. G.; McLean, M. A.; Shaw, A. W.; Grinkova, Y. V.; Sligar, S. G. J. Phys. Chem. B 2005, 109, 15580.

15) Li, L.; Lin, M. Y.; Qiu, F.; Yang, Y. L. Acta Chim. Sinica 2005, 63, 1375.

16) Pornpattananangkul, D.; Olson, S.; Aryal, S.; Sartor, M.; Huang, C. M.; Vecchio, K.; Zhang, L. ACS Nano 2010, 4, 1935. 
17) Roux, M.; Moutard, S.; Perly, B.; Djedaini-Pilard, F. Biophys. J. 2007, 93, 1620.

18) Ginzburg, V. V.; Balijepalli, S. Nano Lett. 2007, 7, 3716.

19) Binder, W. H. Angew. Chem. Int. Ed. 2008, 47, 3092.

20) Wang, B.; Zhang, L. F.; Bae, S. C.; Granick, S. Proc. Natl. Acad. Sci. USA 2008, 105, 18171.

21) Binder, W. H.; Sachsenhofer, R.; Farnik, D.; Blaas, D. Phys. Chem. Chem. Phys. 2007, 9 , 6435.

22) Fang, C.; Fan, Y.; Kong, J. M.; Gao, Z. Q.; Balasubramanian, N. Appl. Phys. Lett. 2008, 92, 2631081.

23) Brust, M.; Walker, M.; Bethell, D.; Schiffrin, D. J.; Whyman, R. J. Chem. Soc., Chem. Commun. 1994, 801.

24) Smetana, A. B.; Wang, J. S.; Boeckl, J.; Brown, G. J.; Wai, C. M. Langmuir, 2007, 23, 20429.

25) Leroueil, P. R.; Berry,S. A.; Duthie, K.; Han, G.; Rotello, V. M.; McNerny, D. Q.; Baker, J. R.; Orr, B. G.; Holl, M. M. Nano Lett. 2008, 8, 420.

26) Vivares, E.; Ramos, L. Langmuir 2005, 21, 2185.

27) Cambrea, L. R.; Hovis, J. S. Biophys. J. 2007, 92, 3587.

28) Seu, K. J.; Lamberson, E. R.; Hovis, J. S. J. Phys. Chem. B 2007, 111, 6289.

29) Goertz, M. P.; Goyal, N.; Montano, G. A.; Bunker, B. C. Langmuir 2011, 27,5481-5491.

30) Willets, K. A.; Van Duyne, R. P. Ann.Rev. Phys. Chem. 2007, 58, 267.

31) Haes, A. J.; Van Duyne, R. P. Anal. Bioanal. Chem. 2004, 379, 920.

32) Solovkin, A. S.; Tsvetkova, S. V. Russian Chem. Rev. 1962, 31, 655.

33) Fay, R. C. in Wilkinson, G.; Gillard, R. D.; and McCleverty, J. A. (Eds) Comprehensive Coordination Chemistry, Pergamon Press, Oxford, 1987, Ch.3.

34)Willey, G. R.; Woodman, T. J.; Fishes, M. TRansition Met. Chem. 1998, 23, 467.

35) Shervedani, R. K.; Bagherzadeh, M. Biosens. Bioelectron. 2009, 24, 2199.

36) Larsson, E. M.; Alegret, J.; Kall, M.; Sutherland, D. S. Nano Lett. 2007, 7, 1256.

37) Sun, Q.; Wang, Q.; Jena, P.; Note, R.; Yu, J. Z.; Kawazoe, Y. Phys. Rev. B 2004, 70, 245411-1.

38) Aizpura, J.; Hanarp, P.; Sutherland, D. S.; Kall, M.; Bryant, G. W.; de Abajo, F. J. G. Phys. Rev. Lett. 2003, 90, 054701-1. 


\section{DISTRIBUTION LiST}

$1 \quad$ MS 0899

Technical Library, 9536 (electronic copy)

$1 \quad$ MS 0359

Donna Chavez, LDRD office, 1911 


\section{Sandia National Laboratories}

